\title{
Harmonic Analysis on the Proper Velocity gyrogroup *
}

\author{
Milton Ferreira \\ School of Technology and Management, \\ Polytechnic Institute of Leiria, Portugal \\ 2411-901 Leiria, Portugal. \\ Email: milton.ferreira@ipleiria.pt \\ and
}

Center for Research and Development in Mathematics and Applications (CIDMA), University of Aveiro, 3810-193 Aveiro, Portugal.

Email mferreira@ua.pt

\begin{abstract}
In this paper we study harmonic analysis on the Proper Velocity (PV) gyrogroup using the gyrolanguage of analytic hyperbolic geometry. PV addition is the relativistic addition of proper velocities in special relativity and it is related with the hyperboloid model of hyperbolic geometry. The generalized harmonic analysis depends on a complex parameter $z$ and on the radius $t$ of the hyperboloid and comprises the study of the generalized translation operator, the associated convolution operator, the generalized Laplace-Beltrami operator and its eigenfunctions, the generalized Poisson transform and its inverse, the generalized Helgason-Fourier transform, its inverse and Plancherel's Theorem. In the limit of large $t, t \rightarrow+\infty$, the generalized harmonic analysis on the hyperboloid tends to the standard Euclidean harmonic analysis on $\mathbb{R}^{n}$, thus unifying hyperbolic and Euclidean harmonic analysis.
\end{abstract}

Keywords: PV gyrogroup, Laplace Beltrami operator, Eigenfunctions, Generalized HelgasonFourier transform, Plancherel's Theorem.

\section{Introduction}

Harmonic analysis is the branch of mathematics that studies the representation of functions or signals as the superposition of basic waves called harmonics. It investigates and generalizes the notions of Fourier series and Fourier transforms. In the past two centuries, it has become a vast subject with applications in diverse areas as signal processing, quantum mechanics, and neuroscience (see [18] for an overview).

Noncommutative harmonic analysis appeared mainly in the context of symmetric spaces where many Lie groups are locally compact and noncommutative. These examples are of interest and frequently applied in mathematical physics and contemporary number theory, particularly automorphic representations. The development of noncommutative harmonic analysis was done by many mathematicians among which stand out the names of John von Neumann, Harisch-Chandra and Sigurdur Helgason [10, 11, 12].

Fourier analysis on real Euclidean space is intimately connected with the action of the group of translations. The group structure enters into the study of harmonic analysis by

\footnotetext{
*Accepted author's manuscript (AAM) published in [Banach J. Math. Anal., 11(1) (2017), 21-49]. The final publication is available at http://projecteuclid.org/euclid.bjma/1476841712.
} 
allowing the consideration of the translates of the object under study (functions, measures, etc.). On real hyperbolic space the same approach can be done considering the gyrogroup structure underlying hyperbolic space. In our recent papers $[5,6]$ we developed generalized harmonic analysis on Möbius and Einstein gyrogroups which are related to the Poincaré and Klein-Beltrami models of hyperbolic geometry. The gyrogroup structure is a natural extension of the group structure, discovered in 1988 by A. A. Ungar in the context of Einstein's velocity addition law [19]. It has been extensively studied by A. A. Ungar and others see, for instance, [7, 20, 21, 23]. Gyrogroups provide a fruitful bridge between nonassociative algebra and hyperbolic geometry, just as groups lay the bridge between associative algebra and Euclidean geometry. Our aim in this paper is to present new results connecting harmonic analysis on the hyperboloid model of real hyperbolic space and its gyrogroup structure. This model is algebraically regulated by the Proper Velocity (PV) addition of proper velocities in special relativity. PV addition plays a similar role to that of vector addition in the Euclidean $n$-space $\mathbb{R}^{n}$ giving rise to the PV gyrogroup $[7,21,22]$. Proper time is useful, for instance in the understanding of the twin paradox and the mean life time of unstable moving particles. In quantum mechanics it is useful to reformulate relativity physics in terms of proper time instead of coordinate time (see [9]).

In this paper we study several aspects of harmonic analysis on the PV gyrogroup associated to the family of Laplace-Beltrami operators $\Delta_{z, t}$ in $\mathbb{R}^{n}$ given by

$$
\Delta_{z, t}=\Delta+\sum_{i, j=1}^{n} \frac{x_{i} x_{j}}{t^{2}} \frac{\partial^{2}}{\partial x_{i} \partial x_{j}}+(n-2 z) \sum_{i=1}^{n} \frac{x_{i}}{t^{2}} \frac{\partial}{\partial x_{i}}+\frac{z(z+1)}{t^{2}}\left(1-\beta_{x}^{2}\right)
$$

where $z \in \mathbb{C}, t \in \mathbb{R}^{+}$, and $\beta_{x}$ is the relativistic beta factor. Eigenfunctions of this operator are parameterized by the eigenvalues $-\lambda^{2}-\frac{(n-1)^{2}}{4 t^{2}}+\frac{n z}{t^{2}}$, with $\lambda \in \mathbb{C}$. For $z=0$ and the normalized case $t=1$ we obtain the eigenvalues of the common Laplace-Beltrami operator on the hyperboloid model considered e.g. in $[2,17]$. Our approach extends some of the results of these papers. Possible applications of our work comprises the study of generalized coherent states on hyperbolic space and hamiltonian systems. In [1] the authors proposed a Wigner quasiprobability distribution function for Hamiltonian systems on hyperboloids based on the eigenfunctions of the Laplace-Beltrami on the hyperboloid.

The paper is organized as follows. In Section 2 we present the PV addition and its properties. Sections 3 and 4 are dedicated to the study of the generalized translation operator and the associated convolution operator. In Section 5 we construct the eigenfunctions of the generalized Laplace-Beltrami operator on the hyperboloid and we study the generalized spherical functions. In Section 6 we define the generalized Poisson transform and we study the injectivity of this transform. Section 7 is devoted to the generalized Helgason Fourier transform on the PV gyrogroup. In Section 8 we obtain the inversion formula for the generalized Helgason Fourier transform and Plancherel's Theorem and we show that in the limit $t \rightarrow+\infty$ we recover the inverse Fourier transform and Plancherel's Theorem in Euclidean harmonic analysis. As an application, in Section 9 we solve the heat equation on the proper velocity gyrogroup. Two appendices, A and B, concerning all necessary facts on spherical harmonics and Jacobi functions, are found at the end of the paper.

\section{Proper Velocity addition}

Proper velocities in special relativity theory are velocities measured by proper time, that is, by travelerâẮ́s time rather than by observerâẮ́s time [3]. The addition of proper velocities was defined by A.A. Ungar in [3] giving rise to the proper velocity gyrogroup. 
Definition 2.1. [21] Let $(V,+,\langle\rangle$,$) be a real inner product space with addition +$, and inner product $\langle$,$\rangle . The PV (Proper Velocity) gyrogroup (V, \oplus)$ is the real inner product space $V$ equipped with addition $\oplus$ given by

$$
a \oplus x=x+\left(\frac{\beta_{a}}{1+\beta_{a}} \frac{\langle a, x\rangle}{t^{2}}+\frac{1}{\beta_{x}}\right) a
$$

where $t \in \mathbb{R}^{+}$and $\beta_{a}$, called the relativistic beta factor, is given by the equation

$$
\beta_{a}=\frac{1}{\sqrt{1+\frac{\|a\|^{2}}{t^{2}}} .}
$$

$\mathrm{PV}$ addition is the relativistic addition of proper velocities rather than coordinate velocities as in Einstein addition. PV addition satisfies the beta identity

$$
\beta_{a \oplus x}=\frac{\beta_{a} \beta_{x}}{1+\beta_{a} \beta_{x} \frac{\langle a, x\rangle}{t^{2}}}
$$

or, equivalently,

$$
\frac{\beta_{x}}{\beta_{a \oplus x}}=\frac{1}{\beta_{a}}+\beta_{x} \frac{\langle a, x\rangle}{t^{2}} .
$$

It is known that $(V, \oplus)$ is a gyrogroup (see [21]), i.e. it satisfies the following axioms:

(G1) There is at least one element 0 satisfying $0 \oplus a=a$, for all $a \in V$;

(G2) For each $a \in V$ there is an element $\ominus a \in V$ such that $\ominus a \oplus a=0$;

(G3) For any $a, b, c \in V$ there exists a unique element $\operatorname{gyr}[a, b] c \in V$ such that the binary operation satisfies the left gyroassociative law

$$
a \oplus(b \oplus c)=(a \oplus b) \oplus \operatorname{gyr}[a, b] c
$$

(G4) The map gyr $[a, b]: V \rightarrow V$ given by $c \mapsto \operatorname{gyr}[a, b] c$ is an automorphism of $(V, \oplus)$;

(G5) The gyroautomorphism gyr $[a, b]$ possesses the left loop property

$$
\operatorname{gyr}[a, b]=\operatorname{gyr}[a \oplus b, b] .
$$

PV gyrations can be given in terms of the PV addition $\oplus$ by the equation (see [21])

$$
\operatorname{gyr}[a, b] c=\ominus(a \oplus b) \oplus(a \oplus(b \oplus c)) .
$$

The PV gyrogroup is gyrocommutative since PV addition satisfies

$$
a \oplus b=\operatorname{gyr}[a, b](b \oplus a) .
$$

In the limit $t \rightarrow+\infty$, PV addition reduces to vector addition in $(V,+)$ and, therefore, the gyrogroup $(V, \oplus)$ reduces to the translation group $(V,+)$. To see the connection between proper velocity addition, proper Lorentz transformations, and real hyperbolic geometry let us consider the one sheeted hyperboloid $H_{t}^{n}=\left\{x \in \mathbb{R}^{n+1}: x_{n+1}^{2}-x_{1}^{2}-\ldots-x_{n}^{2}=t^{2} \wedge x_{n+1}>0\right\}$ in $\mathbb{R}^{n+1}$ where $t \in \mathbb{R}^{+}$is the radius of the hyperboloid. The $n$-dimensional real hyperbolic space is usually viewed as the rank one symmetric space $G / K$ of noncompact type, where $G=\operatorname{SO}_{e}(n, 1)$ is the identity connected component of the group of orientation preserving isometries of $H_{t}^{n}$ 
and $K=\mathrm{SO}(n)$ is the maximal compact subgroup of $G$ which stabilizes the base point $O:=(0, \ldots, 0,1)$ in $\mathbb{R}^{n+1}$. Thus, $H_{t}^{n} \cong \mathrm{SO}_{e}(1, n) / \mathrm{SO}(n)$ and it is one model for real hyperbolic geometry with constant negative curvature. Restricting the semi-Riemannian metric $d x_{n+1}^{2}-d x_{1}^{2}-\ldots-d x_{n}^{2}$ on the ambient space we obtain the Riemannian metric on $H_{t}^{n}$ which is given by

$$
d s^{2}=\frac{(\langle x, d x\rangle)^{2}}{t^{2}+\|x\|^{2}}-\|d x\|^{2}
$$

with $x=\left(x_{1}, \ldots, x_{n}\right) \in \mathbb{R}^{n}$ and $d x=\left(d x_{1}, \ldots, d x_{n}\right)$. This metric corresponds to the metric tensor

$$
g_{i j}(x)=\frac{x_{i} x_{j}}{t^{2}+\|x\|^{2}}-\delta_{i j}, \quad i, j \in\{1, \ldots, n\}
$$

whereas the inverse metric tensor is given by

$$
g^{i j}(x)=-\delta_{i j}-\frac{x_{i} x_{j}}{t^{2}}, \quad i, j \in\{1, \ldots, n\} .
$$

The group of all orientation preserving isometries of $H_{t}^{n}$ consists of elements of the group $\mathrm{SO}(n)$ and proper Lorentz transformations acting on $H_{t}^{n}$. A simple way of working in $H_{t}^{n}$ is to consider its projection into $\mathbb{R}^{n}$. Given an arbitray point $\left(x, \sqrt{t^{2}+\|x\|^{2}}\right) \in H_{t}^{n}$ we define the mapping $\Pi: H_{t}^{n} \rightarrow \mathbb{R}^{n}$, such that $\Pi\left(x, \sqrt{t^{2}+\|x\|^{2}}\right)=x$.

A proper Lorentz boost in the direction $\omega \in S^{n-1}$ and rapidity $\alpha$ acting in an arbitrary point $\left(x, \sqrt{t^{2}+\|x\|^{2}}\right) \in H_{t}^{n}$ yields a new point $\left(x, x_{n+1}\right)_{\omega, \alpha} \in H_{t}^{n}$ given by (see [4])

$$
\begin{aligned}
\left(x, x_{n+1}\right)_{\omega, \alpha}= & \left(x+\left((\cosh (\alpha)-1)\langle\omega, x\rangle-\sinh (\alpha) \sqrt{t^{2}+\|x\|^{2}}\right) \omega\right. \\
& \left.\cosh (\alpha) \sqrt{t^{2}+\|x\|^{2}}-\sinh (\alpha)\langle\omega, x\rangle\right) .
\end{aligned}
$$

Since

$$
\sqrt{t^{2}+\left\|x+\left((\cosh (\alpha)-1)\langle\omega, x\rangle-\sinh (\alpha) \sqrt{t^{2}+\|x\|^{2}}\right) \omega\right\|^{2}}=x_{n+1}
$$

then the projection of (6) into $\mathbb{R}^{n}$ is given by

$$
\Pi\left(x, x_{n+1}\right)_{\omega, \alpha}=x+\left((\cosh (\alpha)-1)\langle\omega, x\rangle-\sinh (\alpha) \sqrt{t^{2}+\|x\|^{2}}\right) \omega .
$$

Rewriting the parameters of the Lorentz boost to depend on a point $a \in \mathbb{R}^{n}$ as

$$
\cosh (\alpha)=\sqrt{1+\frac{\|a\|^{2}}{t^{2}}}, \quad \sinh (\alpha)=-\frac{\|a\|}{t}, \quad \text { and } \quad \omega=\frac{a}{\|a\|} .
$$

and replacing (8) in (7) we finally obtain the relativistic addition of proper velocities in $\mathbb{R}^{n}$ :

$$
\begin{aligned}
a \oplus x & =x+\left(\frac{\sqrt{1+\frac{\|a\|^{2}}{t^{2}}}-1}{\|a\|^{2}}\langle a, x\rangle+\sqrt{1+\frac{\|x\|^{2}}{t^{2}}}\right) a \\
& =x+\left(\frac{\beta_{a}}{1+\beta_{a}} \frac{\langle a, x\rangle}{t^{2}}+\frac{1}{\beta_{x}}\right) a
\end{aligned}
$$

From now on we consider the PV gyrogroup $\left(\mathbb{R}^{n}, \oplus\right)$. In this case $\ominus a=-a$. For $R \in \operatorname{SO}(n)$ we have the homomorphism

$$
R(a \oplus x)=(R a) \oplus(R x)
$$


Some useful gyrogroup identities ([21], pp. 48 and 68) that will be used in this paper are

$$
\begin{gathered}
\operatorname{gyr}[a, b])^{-1}=\operatorname{gyr}[b, a] \\
\operatorname{gyr}[a \oplus b, \ominus a]=\operatorname{gyr}[a, b] \\
\operatorname{gyr}[\ominus a, \ominus b]=\operatorname{gyr}[a, b] \\
\operatorname{gyr}[a, b](b \oplus(a \oplus c))=(a \oplus b) \oplus c
\end{gathered}
$$

and (12) are valid only for gyrocommutative gyrogroups. Combining formulas (10) and (12) with (9) we obtain the identities

$$
\begin{gathered}
\operatorname{gyr}[\ominus a, a \oplus b]=\operatorname{gyr}[b, a] \\
b \oplus(a \oplus c)=\operatorname{gyr}[b, a]((a \oplus b) \oplus c) .
\end{gathered}
$$

For $n \geq 2$ the gyrosemidirect product of $(\mathbb{R}, \oplus)$ and $\mathrm{SO}(n)$ (see [21]) gives the group $\mathbb{R} \rtimes_{\text {gyr }} \mathrm{SO}(n)$ for the operation

$$
(a, R)(b, S)=(a \oplus R b, \operatorname{gyr}[a, R b] R S) .
$$

This group is a realization of the Lorentz group $\mathrm{SO}_{e}(1, n)$. In the limit $t \rightarrow+\infty$ the group $\mathbb{R} \rtimes_{\text {gyr }} \mathrm{SO}(n)$ reduces to the Euclidean group $\mathrm{E}(n)=\mathbb{R}^{n} \rtimes \mathrm{SO}(n)$. This shows that $\left(\mathbb{R}^{n}, \oplus\right)$ is the appropriate algebraic structure to develop harmonic analysis on the hyperboloid.

\section{The generalized translation}

Definition 3.1. For a function $f$ defined on $\mathbb{R}^{n}, a \in \mathbb{R}^{n}$, and $z \in \mathbb{C}$ we define the generalized translation operator $\tau_{a} f$ by the complex-valued function

$$
\tau_{a} f(x)=j_{a}(x) f((-a) \oplus x)
$$

with

$$
j_{a}(x)=\left(\frac{\beta_{a}}{1-\beta_{a} \beta_{x} \frac{\langle a, x\rangle}{t^{2}}}\right)^{z} .
$$

The multiplicative factor $j_{a}(x)$ agrees with the Jacobian of the transformation $(-a) \oplus x$ when $z=1$. In the case $z=0$ the translation reduces to $\tau_{a} f(x)=f((-a) \oplus x)$. Moreover, for any $z \in \mathbb{C}$, we obtain in the limit $t \rightarrow+\infty$ the Euclidean translation operator $\tau_{a} f(x)=$ $f(-a+x)=f(x-a)$. By (14) and (3) we can write $j_{a}(x)$ as $j_{a}(x)=\left(\frac{\beta_{(-a) \oplus x}}{\beta_{x}}\right)^{z}$.

Lemma 3.2. For any $a, b, x, y \in \mathbb{R}^{n}$ the following relations hold

$$
\begin{aligned}
(i) & j_{-a}(-x)=j_{a}(x) \\
(i i) & j_{a}(a) j_{a}(0)=1 \\
(i i i) & j_{a}(x)=j_{x}(a) j_{a}(0) j_{x}(x) \\
(i v) & j_{a}(a \oplus x)=\left(j_{-a}(x)\right)^{-1} \\
(v) & j_{(-a) \oplus x}(0)=j_{x \oplus(-a)}(0)=j_{x}(a) j_{a}(0)=j_{a}(x) j_{x}(0) \\
(v i) & j_{(-a) \oplus x}((-a) \oplus x)=\left(j_{a}(x)\right)^{-1} j_{x}(x) \\
(v i i) & \tau_{a} j_{y}(x)=\left[\tau_{-a} j_{x}(y)\right] j_{x}(x) j_{y}(0) \\
(v i i i) & \tau_{-a} j_{a}(x)=1 \\
(\text { ix }) & \tau_{a} j_{y}(x)=j_{a \oplus y}(x) \\
(x) & \tau_{a} f(x)=\left[\tau_{x} f(-\operatorname{gyr}[x, a] a)\right] j_{a}(0) j_{x}(x) \\
(x i) & \tau_{b} \tau_{a} f(x)=\tau_{b \oplus a} f(\operatorname{gyr}[a, b] x) \\
(x i i) & \tau_{-a} \tau_{a} f(x)=f(x) \\
(x i i i) & \tau_{b} \tau_{a} f(x)=\left[\tau_{-b} \tau_{x} f(-\operatorname{gyr}[-b, x \oplus a] \operatorname{gyr}[x, a] a)\right] j_{a}(0) j_{x}(x) .
\end{aligned}
$$


The proof of these relations uses the beta-identity (3) and the same techniques as in the Einstein case (see [5]). To compute the generalized Laplace-Beltrami operator $\Delta_{z, t}$ that commutes with the generalized translation operator (13) we use the approach of Rudin $[16$, Ch. 4$]$ and we compute $\Delta\left(\tau_{-a} f\right)(0)$, where $\Delta$ is the Laplace operator in $\mathbb{R}^{n}$.

Proposition 3.3. For each $f \in C^{2}\left(\mathbb{R}^{n}\right)$ and $a \in \mathbb{R}^{n}$

$$
\begin{aligned}
\Delta\left(\tau_{-a} f\right)(0)= & j_{a}(0)\left(\Delta f(a)+\sum_{i, j=1}^{n} \frac{a_{i} a_{j}}{t^{2}} \frac{\partial^{2} f}{\partial x_{i} \partial x_{j}}(a)+(n-2 z) \sum_{i=1}^{n} \frac{a_{i}}{t^{2}} \frac{\partial f}{\partial x_{i}}(a)+\right. \\
& \left.+\frac{z(z+1)}{t^{2}}\left(1-\beta_{a}^{2}\right) f(a)\right) .
\end{aligned}
$$

Proof. Let $a \in \mathbb{R}^{n}$ and denote by $T_{1}, \ldots, T_{n}$ the coordinates of the mapping $a \oplus x$. Then by the chain rule we have

$$
\begin{aligned}
\Delta\left(\tau_{-a} f\right)(0)= & {\left[\sum_{j, k=1}^{n} \frac{\partial^{2} f}{\partial x_{j} \partial x_{k}}(a) \sum_{i=1}^{n} \frac{\partial T_{k}}{\partial x_{i}}(0) \frac{\partial T_{j}}{\partial x_{i}}(0)+\sum_{k=1}^{n} \frac{\partial f}{\partial x_{k}}(a) \sum_{i=1}^{n} \frac{\partial^{2} T_{k}}{\partial x_{i}^{2}}(0)\right] j_{-a}(0) } \\
& +2 \sum_{k=1}^{n} \frac{\partial f}{\partial x_{k}}(a) \sum_{i=1}^{n} \frac{\partial T_{k}}{\partial x_{i}}(0) \frac{\partial j_{-a}}{\partial x_{i}}(0)+f(a) \sum_{i=1}^{n} \frac{\partial^{2} j_{-a}}{\partial x_{i}^{2}}(0) .
\end{aligned}
$$

Since $T_{k}(x)=x_{k}+\left(\frac{\beta_{a}}{1+\beta_{a}} \frac{\langle a, x\rangle}{t^{2}}+\frac{1}{\beta_{x}}\right) a_{k}, k \in\{1, \ldots, n\}$ then

$$
\frac{\partial T_{k}}{\partial x_{i}}(0)=\delta_{k, i}+\frac{\beta_{a}}{1+\beta_{a}} \frac{a_{i} a_{k}}{t^{2}} \quad \text { and } \quad \frac{\partial^{2} T_{k}}{\partial x_{i}^{2}}(0)=\frac{a_{k}}{t^{2}}, \quad i, k \in\{1, \ldots, n\} .
$$

Moreover,

$$
\frac{\partial j_{-a}}{\partial x_{i}}(0)=-j_{a}(0) z \beta_{a} \frac{a_{i}}{t^{2}}
$$

and

$$
\frac{\partial^{2} j_{-a}}{\partial x_{i}^{2}}(0)=j_{a}(0) \frac{z(z+1)}{t^{2}} \beta_{a}^{2} \frac{a_{i}^{2}}{t^{2}} .
$$

Therefore, replacing these expressions in (29) and after straightforward computations we obtain (28).

Definition 3.4. Let $\Omega$ be an open subset of $\mathbb{R}^{n}$ and $f \in C^{2}(\Omega)$. We define the generalized Laplace Beltrami-operator $\Delta_{z, t}$ on $H_{t}^{n}$ by

$$
\Delta_{z, t}=\Delta+\sum_{i, j=1}^{n} \frac{x_{i} x_{j}}{t^{2}} \frac{\partial^{2}}{\partial x_{i} \partial x_{j}}+(n-2 z) \sum_{i=1}^{n} \frac{x_{i}}{t^{2}} \frac{\partial}{\partial x_{i}}+\frac{z(z+1)}{t^{2}}\left(1-\beta_{x}^{2}\right) .
$$

By Proposition 3.3 we obtain a representation formula for $\Delta_{z, t}$ :

$$
\Delta_{z, t} f(a)=\left(j_{a}(0)\right)^{-1} \Delta\left(\tau_{-a} f\right)(0) .
$$

Proposition 3.5. The operator $\Delta_{z, t}$ commutes with generalized translations, i.e.

$$
\Delta_{z, t}\left(\tau_{b} f\right)=\tau_{b}\left(\Delta_{z, t} f\right) \quad \forall f \in C^{2}\left(\mathbb{R}^{n}\right), \forall b \in \mathbb{R}^{n} .
$$


Proof. By (30) we have

$$
\begin{aligned}
\Delta_{z, t}\left(\tau_{b} f\right)(a) & =\left(j_{a}(0)\right)^{-1} \Delta\left(\tau_{-a} \tau_{b} f\right)(0) \\
& =\left.\left(j_{a}(0)\right)^{-1} \Delta\left(f((-b) \oplus(a \oplus x)) \tau_{-a} j_{b}(x)\right)\right|_{x=0} .
\end{aligned}
$$

Now, since

$$
(-b) \oplus(a \oplus x)=((-b) \oplus a) \oplus \operatorname{gyr}[-b, a] x \quad \text { (by }(4))
$$

and

$$
\begin{array}{rlrl}
\tau_{-a} j_{b}(x) & =j_{(-a) \oplus b}(x) \quad(\text { by }(23)) & \\
& =j_{b \oplus(-a)}(\operatorname{gyr}[b,-a] x) & & (\text { by }(5),(9)) \\
& =j_{b \oplus(-a)}(\operatorname{gyr}[-b, a] x) & & (\text { by }(11))
\end{array}
$$

then together with the invariance of $\Delta$ under the group $\mathrm{SO}(n),(19)$ and (15) we obtain

$$
\begin{aligned}
\Delta_{z, t}\left(\tau_{b} f\right)(a) & =\left.\left(j_{a}(0)\right)^{-1} \Delta\left(f(((-b) \oplus a) \oplus \operatorname{gyr}[-b, a] x) j_{b \oplus(-a)}(\operatorname{gyr}[-b, a] x)\right)\right|_{x=0} \\
& =\left(j_{a}(0)\right)^{-1} \Delta\left(\tau_{-((-b) \oplus a)} f\right)(0) \\
& =j_{b}(a)\left(j_{(-b) \oplus a}(0)\right)^{-1} \Delta\left(\tau_{-((-b) \oplus a)} f\right)(0) \\
& =j_{b}(a)\left(\Delta_{z, t} f\right)((-b) \oplus a) \\
& =\tau_{b}\left(\Delta_{z, t} f\right)(a) .
\end{aligned}
$$

For studying some $L^{2}$-properties of the invariant Laplace $\Delta_{z, t}$ and the generalized translation we consider the weighted Hilbert space $L^{2}\left(\mathbb{R}^{n}, d \mu_{z, t}\right)$ with

$$
d \mu_{z, t}(x)=\left(\beta_{x}\right)^{2 z+1} d x=\left(1+\frac{\|x\|^{2}}{t^{2}}\right)^{-\frac{2 z+1}{2}} d x,
$$

where $d x$ stands for the Lebesgue measure in $\mathbb{R}^{n}$. For the special case $z=0$ we recover the invariant measure associated to $a \oplus x$.

Proposition 3.6. For $f, g \in L^{2}\left(\mathbb{R}^{n}, d \mu_{z, t}\right)$ and $a \in \mathbb{R}^{n}$ we have

$$
\int_{\mathbb{R}^{n}} \tau_{a} f(x) \overline{g(x)} d \mu_{z, t}(x)=\int_{\mathbb{R}^{n}} f(x) \overline{\tau_{-a} g(x)} d \mu_{z, t}(x) .
$$

Corollary 3.7. For $f, g \in L^{2}\left(\mathbb{R}^{n}, d \mu_{z, t}\right)$ and $a \in \mathbb{R}^{n}$ we have

$$
\begin{aligned}
& \text { (i) } \int_{\mathbb{R}^{n}} \tau_{a} f(x) d \mu_{z, t}(x)=\int_{\mathbb{R}^{n}} f(x) j_{-a}(x) d \mu_{z, t}(x) ; \\
& \text { (ii) If } z=0 \text { then } \int_{\mathbb{R}^{n}} \tau_{a} f(x) d \mu_{z, t}(x)=\int_{\mathbb{R}^{n}} f(x) d \mu_{z, t}(x) ; \\
& \text { (iii) }\left\|\tau_{a} f\right\|_{2}=\|f\|_{2} .
\end{aligned}
$$

From Corollary 3.7 we see that the generalized translation $\tau_{a}$ is an unitary operator in $L^{2}\left(\mathbb{R}^{n}, d \mu_{z, t}\right)$ and the measure $d \mu_{z, t}$ is translation invariant only for the case $z=0$. There is an important relation between the operator $\Delta_{z, t}$ and the measure $d \mu_{z, t}$. Up to a constant the Laplace-Beltrami operator $\Delta_{z, t}$ corresponds to a weighted Laplace operator on $\mathbb{R}^{n}$ for the weighted measure $d \mu_{z, t}$ in the sense defined in [8, Sec. 3.6]. From Theorem 11.5 in [8] we know that the Laplace operator on a weighted manifold is essentially self-adjoint if all geodesics balls are relatively compact. Therefore, $\Delta_{z, t}$ can be extended to a self adjoint operator in $L^{2}\left(\mathbb{R}^{n}, d \mu_{z, t}\right)$.

Proposition 3.8. The operator $\Delta_{z, t}$ is essentially self-adjoint in $L^{2}\left(\mathbb{R}^{n}, d \mu_{z, t}\right)$. 


\section{The generalized convolution}

In this section we define the generalized convolution of two functions, we study its properties and we establish the respective Young's inequality and gyroassociative law. In the limit $t \rightarrow+\infty$ both definitions and properties tend to their Euclidean counterparts.

Definition 4.1. The generalized convolution of two measurable functions $f$ and $g$ is given by

$$
(f * g)(x)=\int_{\mathbb{R}^{n}} f(y) \tau_{x} g(-y) j_{x}(x) d \mu_{z, t}(y), \quad x \in \mathbb{R}^{n} .
$$

By Proposition 3.6 we have

$$
\begin{aligned}
(f * g)(x) & =\int_{\mathbb{R}^{n}} \tau_{-x} f(y) g(-y) j_{x}(x) d \mu_{z, t}(y) \\
& =\int_{\mathbb{R}^{n}} f(x \oplus y) j_{-x}(y) g(-y) j_{x}(x) d \mu_{z, t}(y) \quad(\text { by } \quad(13)) \\
& =\int_{\mathbb{R}^{n}} f(x \oplus(-y)) j_{-x}(-y) g(y) j_{x}(x) d \mu_{z, t}(y) \quad(y \mapsto-y) \\
& =\int_{\mathbb{R}^{n}} \tau_{x} f(-y) g(y) j_{x}(x) d \mu_{z, t}(y) \quad(\text { by }(15),(13)) \\
& =(g * f)(x) .
\end{aligned}
$$

Thus, the generalized convolution is commutative.

Lemma 4.2. Let $\Re(z)<\frac{n-1}{2}$. Then for any $t>0$ and $s>0$ we have

$$
\int_{\mathbb{S}^{n-1}}\left|j_{x}(t \sinh (s) \xi) j_{x}(x)\right| d \sigma(\xi) \leq C_{z}
$$

with

$$
C_{z}=\left\{\begin{array}{cl}
1, & \text { if } \Re(z) \in]-1,0[ \\
\frac{\Gamma\left(\frac{n}{2}\right) \Gamma\left(\frac{n-2 \Re(z)-1}{2}\right)}{\Gamma\left(\frac{n-\Re(z)}{2}\right) \Gamma\left(\frac{n-\Re(z)-1}{2}\right)}, & \text { if } \Re(z) \in]-\infty,-1] \cup\left[0, \frac{n-1}{2}[\right.
\end{array} .\right.
$$

Proof. Using (63) in Appendix A we obtain

$$
\begin{aligned}
\int_{\mathbb{S}^{n-1}}\left|j_{x}(t \sinh (s) \xi) j_{x}(x)\right| d \sigma(\xi) & \\
& =\int_{\mathbb{S}^{n-1}}\left|\left(\frac{\beta_{x}}{1-\frac{\left\langle\beta_{x} x, \beta_{t \sinh (s) \xi} t \sinh (s) \xi\right\rangle}{t^{2}}}\right)^{z}\left(\frac{1}{\beta_{x}}\right)^{z}\right| d \sigma(\xi) \\
& =\int_{S^{n-1}}\left(\frac{1}{1-\frac{\left\langle\tanh (s) \beta_{x} x, \xi\right\rangle}{t}}\right)^{\Re(z)} d \sigma(\xi) \\
& ={ }_{2} F_{1}\left(\frac{\Re(z)}{2}, \frac{\Re(z)+1}{2} ; \frac{n}{2} ; \frac{\tanh ^{2}(s)\left(\beta_{x}\right)^{2}\|x\|^{2}}{t^{2}}\right) .
\end{aligned}
$$

Since $\left(\beta_{x}\right)^{2} \frac{\|x\|^{2}}{t^{2}}=1-\left(\beta_{x}\right)^{2}<1$ we can consider for $r \in[0,1[$ the function $g(r)=$ 
${ }_{2} F_{1}\left(\frac{\Re(z)}{2}, \frac{\Re(z)+1}{2} ; \frac{n}{2} ; r\right)$. Applying (67) and (65) in Appendix A we get

$$
\begin{aligned}
& g^{\prime}(r)=\frac{\Re(z)(\Re(z)+1)}{2 n}{ }_{2} F_{1}\left(\frac{\Re(z)+2}{2}, \frac{\Re(z)+3}{2} ; \frac{n}{2}+1 ; r\right) . \\
& =\underbrace{\frac{\Re(z)(\Re(z)+1)}{2 n}}_{(I)}(1-r)^{\frac{n-2 \Re(z)-3}{2}} \underbrace{{ }_{2} F_{1}\left(\frac{n-\Re(z)}{2}, \frac{n-\Re(z)-1}{2} ; \frac{n}{2}+1 ; r\right)}_{(I I)} .
\end{aligned}
$$

Since $\Re(z)<\frac{n-1}{2}$ then the hypergeometric function $(I I)$ is positive for $r>0$, and therefore, positive on the interval [0,1[. Studying the sign of $(I)$ we conclude that the function $g$ is strictly increasing when $\Re(z) \in]-\infty,-1] \cup\left[0, \frac{n-1}{2}[\right.$ and strictly decreasing when $\Re(z) \in]-1,0\left[\right.$. Since $\Re(z)<\frac{n-1}{2}$, then by (64) it exists the $\operatorname{limit}_{\lim _{r \rightarrow 1^{-}}} g(r)$ which is given by

$$
g(1)=\frac{\Gamma\left(\frac{n}{2}\right) \Gamma\left(\frac{n-2 \Re(z)-1}{2}\right)}{\Gamma\left(\frac{n-\Re(z)}{2}\right) \Gamma\left(\frac{n-\Re(z)-1}{2}\right)} .
$$

Thus,

$$
g(r) \leq \max \{g(0), g(1)\}=C_{z}
$$

with $g(0)=1$.

Proposition 4.3. Let $\Re(z)<\frac{n-1}{2}$ and $f, g \in L^{1}\left(\mathbb{R}^{n}, d \mu_{z, t}\right)$. Then

$$
\|f * g\|_{1} \leq C_{z}\|f\|_{1}\|\widetilde{g}\|_{1}
$$

where $\widetilde{g}(s)=\operatorname{ess~sup}_{\xi \in \mathbb{S}^{n-1}, y \in \mathbb{R}^{n}} g(\operatorname{gyr}[y, t \sinh (s) \xi] t \sinh (s) \xi)$ for any $s, t>0$.

Proof. Using (24), (16), and (32) we have

$$
\begin{aligned}
I & =\int_{\mathbb{R}^{n}}\left|\tau_{x} g(-y) j_{x}(x)\right| d \mu_{z, t}(x) \\
& =\int_{\mathbb{R}^{n}}\left|\tau_{y} g(\operatorname{gyr}[y, x] x) j_{x}(0) j_{y}(y) j_{x}(x)\right| d \mu_{z, t}(x) \\
& =\int_{\mathbb{R}^{n}}\left|g(\operatorname{gyr}[y, x] x) j_{-y}(x) j_{y}(y)\right| d \mu_{z, t}(x) .
\end{aligned}
$$

Using polar coordinates $x=t \sinh (s) \xi$, with $t, s>0, \xi \in \mathbb{S}^{n-1}$, and the normalised surface area $d \sigma(\xi)=d \xi / A_{n-1}$, with $A_{n-1}$ being the surface area of $\mathbb{S}^{n-1}$ we get

$$
I=A_{n-1} \int_{\mathbb{R}^{+}} \int_{\mathbb{S}^{n-1}}\left|g_{1}(s, y, \xi)\right|\left|j_{-y}(t \sinh (s) \xi) j_{y}(y)\right|(\cosh (s))^{-2 z} t^{n} \sinh (s)^{n-1} d \sigma(\xi) d s .
$$

with $g_{1}(s, y, \xi)=g(\operatorname{gyr}[y, t \sinh (s) \xi] t \sinh (s) \xi)$. For $t>0$ we consider the radial function $\widetilde{g}$ defined by

$$
\widetilde{g}(s)=\operatorname{ess~sup}_{\xi \in \mathbb{S}^{n-1}, y \in \mathbb{R}^{n}} g_{1}(s, y, \xi) .
$$

Therefore, by Lemma 4.2 and (15) we have

$$
\begin{aligned}
I \leq & A_{n-1} \int_{\mathbb{R}^{+}}|\widetilde{g}(s)|(\cosh (s))^{-2 z} t^{n} \sinh (s)^{n-1} d s \\
& \times \int_{\mathbb{S}^{n-1}}\left|j_{-y}(t \sinh (s) \xi) j_{-y}(-y)\right| d \sigma(\xi) \\
\leq & C_{z}\|\widetilde{g}\|_{1} .
\end{aligned}
$$


Finally,

$$
\begin{aligned}
\|f * g\|_{1} & =\int_{\mathbb{R}^{n}}\left|\int_{\mathbb{R}^{n}} f(y) \tau_{x} g(-y) j_{x}(x) d \mu_{z, t}(y)\right| d \mu_{z, t}(x) \\
& \leq \int_{\mathbb{R}^{n}} \int_{\mathbb{R}^{n}}|f(y)|\left|\tau_{x} g(-y) j_{x}(x)\right| d \mu_{z, t}(y) d \mu_{z, t}(x) \\
& =\int_{\mathbb{R}^{n}}|f(y)|\left(\int_{\mathbb{R}^{n}}\left|\tau_{x} g(-y)\right| j_{x}(x) \mid d \mu_{z, t}(x)\right) d \mu_{z, t}(y) \\
& \leq C_{z}\|f\|_{1}\|\widetilde{g}\|_{1} .
\end{aligned}
$$

In the special case when $g$ is a radial function we obtain that $\|f * g\|_{1} \leq C_{z}\|f\|_{1}\|g\|_{1}$ since $\widetilde{g}=g$. We can also prove that for $f \in L^{\infty}\left(\mathbb{R}^{n}, d \mu_{z, t}\right)$ and $g \in L^{1}\left(\mathbb{R}^{n}, d \mu_{z, t}\right)$ we have the inequality

$$
\|f * g\|_{\infty} \leq C_{z}\|\widetilde{g}\|_{1}\|f\|_{\infty} .
$$

By (33), (34), and the Riesz-Thorin interpolation Theorem we further obtain for $f \in$ $L^{p}\left(\mathbb{R}^{n}, d \mu_{z, t}\right)$ and $g \in L^{1}\left(\mathbb{R}^{n}, d \mu_{z, t}\right)$ the inequality

$$
\|f * g\|_{p} \leq C_{z}\|\widetilde{g}\|_{1}\|f\|_{p}
$$

To obtain a Young's inequality for the generalized convolution we restrict ourselves to the case $\Re(z) \leq 0$.

Theorem 4.4. Let $\Re(z) \leq 0,1 \leq p, q, r \leq \infty, \frac{1}{p}+\frac{1}{q}=1+\frac{1}{r}, s=1-\frac{q}{r}, f \in L^{p}\left(\mathbb{R}^{n}, d \mu_{z, t}\right)$ and $g \in L^{q}\left(\mathbb{R}^{n}, d \mu_{z, t}\right)$. Then

$$
\|f * g\|_{r} \leq 2^{-\Re(z)}\|\widetilde{g}\|_{q}^{1-s}\|g\|_{q}^{s}\|f\|_{p}
$$

where $\widetilde{g}(x):=\underset{y \in \mathbb{R}^{n}}{\operatorname{essup}} g(\operatorname{gyr}[y, x] x)$, for any $x \in \mathbb{R}^{n}$.

For the proof we use the following estimate

$$
\left|j_{x}(y) j_{x}(x)\right| \leq 2^{-\Re(z)}, \forall x, y \in \mathbb{R}^{n}, \forall \Re(z) \leq 0 .
$$

and the arguments given in [5].

Corollary 4.5. Let $\Re(z) \leq 0,1 \leq p, q, r \leq \infty, \frac{1}{p}+\frac{1}{q}=1+\frac{1}{r}, f \in L^{p}\left(\mathbb{R}^{n}, d \mu_{z, t}\right)$ and $g \in L^{q}\left(\mathbb{R}^{n}, d \mu_{z, t}\right)$ a radial function. Then,

$$
\|f * g\|_{r} \leq 2^{-\Re(z)}\|g\|_{q}\|f\|_{p}
$$

Remark 1. For $\Re(z)=0$ and taking the limit $t \rightarrow+\infty$ in (35) we recover the Young's inequality for the Euclidean convolution in $\mathbb{R}^{n}$ since in the limit $\widetilde{g}=g$.

Another important property of the Euclidean convolution is the translation invariance and the associativity. In the hyperbolic case it turns out that the convolution is gyro-translation invariant and gyroassociative in the sense of the following theorems (cf. [5] and [6]).

Theorem 4.6. The generalized convolution is gyro-translation invariant:

$$
\tau_{a}(f * g)(x)=\left(\tau_{a} f(\cdot) * g(\operatorname{gyr}[-a, x] \cdot)\right)(x) .
$$


Corollary 4.7. If $g$ is a radial function then the generalized convolution is translation invariant:

$$
\tau_{a}(f * g)=\left(\tau_{a} f\right) * g
$$

Theorem 4.8. If $f, g, h \in L^{1}\left(\mathbb{R}^{n}, d \mu_{z, t}\right)$ then

$$
\left(f *_{a}\left(g *_{x} h\right)\right)(a)=\left(\left(\left(f(x) *_{y} g(\operatorname{gyr}[a,-(y \oplus x)] \operatorname{gyr}[y, x] x)\right)(y)\right) *_{a} h(y)\right)(a) .
$$

Corollary 4.9. If $f, g, h \in L^{1}\left(\mathbb{R}^{n}, d \mu_{z, t}\right)$ and $g$ is a radial function then the generalized convolution is associative. i.e.,

$$
f *(g * h)=(f * g) * h .
$$

From Theorem 4.8 we see that the generalized convolution is associative up to a gyration of the argument of the function $g$. However, if $g$ is a radial function then the corresponding gyration is trivial (that is, it is the identity map) and therefore the generalized convolution becomes associative. Moreover, in the limit $t \rightarrow+\infty$ gyrations reduce to the identity and therefore, the convolution becomes associative in the Euclidean case. If we denote by $L_{R}^{1}\left(\mathbb{R}^{n}, d \mu_{z, t}\right)$ the subspace of $L^{1}\left(\mathbb{R}^{n}, d \mu_{z, t}\right)$ consisting of radial functions then, for $\Re(z)<\frac{n-1}{2}$ the space $L_{R}^{1}\left(\mathbb{R}^{n}, d \mu_{z, t}\right)$ is a commutative associative Banach algebra under the generalized convolution.

\section{Eigenfunctions of $\Delta_{z, t}$}

Definition 5.1. For $\lambda \in \mathbb{C}, \xi \in \mathbb{S}^{n-1}$, and $x \in \mathbb{R}^{n}$ we define the functions $e_{\lambda, \xi ; t}$ by

$$
e_{\lambda, \xi ; t}(x)=\frac{\left(\beta_{x}\right)^{-z+\frac{n-1}{2}+\mathrm{i} \lambda t}}{\left(1-\frac{\left\langle\beta_{x} x, \xi\right\rangle}{t}\right)^{\frac{n-1}{2}+\mathrm{i} \lambda t}} .
$$

The hyperbolic plane waves $e_{\lambda, \xi ; t}(x)$ converge in the limit $t \rightarrow+\infty$ to the Euclidean plane waves $\mathrm{e}^{i\langle x, \lambda \xi\rangle}$. Since

$$
e_{\lambda, \xi ; t}(x)=\left(1-\frac{\left\langle\beta_{x} x, \xi\right\rangle}{t}\right)^{-\frac{n-1}{2}-\mathrm{i} \lambda t}\left(\beta_{x}\right)^{-z+\frac{n-1}{2}+\mathrm{i} \lambda t}
$$

then we obtain

$$
\lim _{t \rightarrow+\infty} e_{\lambda, \xi ; t}(x) \lesssim \lim _{t \rightarrow+\infty}\left[\left(1-\frac{\langle x, \xi\rangle}{t}\right)^{t}\right]^{-i \lambda}=\mathrm{e}^{\mathrm{i}\langle x, \lambda \xi\rangle}
$$

In the Euclidean case the translation of the Euclidean plane waves $\mathrm{e}^{i\langle x, \lambda \xi\rangle}$ decomposes into the product of two plane waves one being a modulation. In the hyperbolic case, the generalized translation of (36) factorizes also in a modulation and the hyperbolic plane wave but it appears a Möbius transformation acting on $\mathbb{S}^{n-1}$ as the next proposition shows.

Proposition 5.2. The translation of $e_{\lambda, \xi ; t}(x)$ admits the factorization

$$
\tau_{a} e_{\lambda, \xi ; t}(x)=j_{a}(0) e_{\lambda, \xi ; t}(-a) e_{\lambda, T_{a}(\xi) ; t}(x)
$$

where

$$
T_{a}(\xi)=\frac{\xi+\frac{a}{t}+\frac{\beta_{a}}{1+\beta_{a}} \frac{\langle a, \xi\rangle a}{t^{2}}}{\frac{1}{\beta_{a}}+\frac{\langle a, \xi\rangle}{t}} .
$$


Proof. We have

$$
\begin{aligned}
& \tau_{a} e_{\lambda, \xi ; t}(x)=e_{\lambda, \xi ; t}((-a) \oplus x) j_{a}(x) \\
& =\frac{\left(\beta_{(-a) \oplus x}\right)^{-z+\frac{n-1}{2}+\mathrm{i} \lambda t}}{\left(1-\frac{\left\langle\beta_{(-a) \oplus x}((-a) \oplus x), \xi\right\rangle}{t}\right)^{\frac{n-1}{2}+\mathrm{i} \lambda t}}\left(\frac{\beta_{a}}{1-\frac{\left\langle\beta_{a} a, \beta_{x} x\right\rangle}{t^{2}}}\right)^{z} \\
& =\left(1-\frac{\beta_{a} \beta_{x}}{1-\beta_{a} \beta_{x} \frac{\langle a, x\rangle}{t^{2}}} \frac{\left(\langle x, \xi\rangle+\frac{\beta_{a}}{1+\beta_{a}} \frac{\langle a, x\rangle\langle a, \xi\rangle}{t^{2}}-\frac{1}{\beta_{x}}\langle a, \xi\rangle\right)}{t}\right)^{-\frac{n-1}{2}-\mathrm{i} \lambda t} \\
& \times\left(\frac{\beta_{a} \beta_{x}}{1-\beta_{a} \beta_{x} \frac{\langle a, x\rangle}{t^{2}}}\right)^{-z+\frac{n-1}{2}+\mathrm{i} \lambda t}\left(\frac{\beta_{a}}{1-\frac{\left\langle\beta_{a} a, \beta_{x} x\right\rangle}{t^{2}}}\right)^{z}, \quad \text { by (1) and (3) } \\
& =\left(1-\beta_{a} \beta_{x} \frac{\langle a, x\rangle}{t^{2}}-\frac{\beta_{a} \beta_{x}}{t}\left(\langle x, \xi\rangle+\frac{\beta_{a}}{1+\beta_{a}} \frac{\langle a, x\rangle\langle a, \xi\rangle}{t^{2}}-\frac{1}{\beta_{x}}\langle a, \xi\rangle\right)\right)^{-\frac{n-1}{2}-\mathrm{i} \lambda t} \\
& \times\left(\beta_{a} \beta_{x}\right)^{-z+\frac{n-1}{2}+\mathrm{i} \lambda t}\left(\beta_{a}\right)^{z} \\
& =\left(\left(1+\frac{\left\langle\beta_{a} a, \xi\right\rangle}{t}\right)\left(1-\frac{\frac{\beta_{a} \beta_{x}}{t}\left\langle x, \frac{a}{t}+\xi+\frac{\beta_{a}}{1+\beta_{a}} \frac{\langle a, \xi\rangle a}{t^{2}}\right\rangle}{1+\beta_{a} \frac{\langle a, \xi\rangle}{t}}\right)\right)^{-\frac{n-1}{2}-\mathrm{i} \lambda t} \\
& \times\left(\beta_{a} \beta_{x}\right)^{-z+\frac{n-1}{2}+\mathrm{i} \lambda t}\left(\beta_{a}\right)^{z} \\
& =\left(\left(1+\frac{\left\langle\beta_{a} a, \xi\right\rangle}{t}\right)\left(1-\frac{1}{t}\left\langle\beta_{x} x, \frac{\frac{a}{t}+\xi+\frac{\beta_{a}}{1+\beta_{a}} \frac{\langle a, \xi\rangle a}{t^{2}}}{\frac{1}{\beta_{a}}+\frac{\langle a, \xi\rangle}{t}}\right\rangle\right)\right)^{-\frac{n-1}{2}-\mathrm{i} \lambda t} \\
& \times\left(\beta_{a} \beta_{x}\right)^{-z+\frac{n-1}{2}+\mathrm{i} \lambda t}\left(\beta_{a}\right)^{z} \\
& =j_{a}(0) e_{\lambda, \xi ; t}(-a) e_{\lambda, T_{a}(\xi)}(x) \text {. }
\end{aligned}
$$

Remark 2. The fractional linear mappings $T_{a}(\xi)$, with $a \in \mathbb{R}^{n}, \xi \in \mathbb{S}^{n-1}$ defined in (39) map the unit sphere $S^{n-1}$ onto itself for any $t>0$ and $a \in \mathbb{R}^{n}$. Moreover, in the limit $t \rightarrow+\infty$ they reduce to the identity mapping on $\mathbb{S}^{n-1}$. It is interesting to observe that the fractional linear mappings obtained from PV addition (1) making the formal substitutions $\frac{x}{t}=\xi$ and $\frac{a \oplus x}{t}=a \oplus \xi$ given by

$$
a \oplus \xi=\xi+\left(\frac{\beta_{a}}{1+\beta_{a}} \frac{\langle a, \xi\rangle}{t}+\sqrt{2}\right) \frac{a}{t}
$$

do not map $S^{n-1}$ onto itself. This is different in comparison with the Möbius and Einstein gyrogroups. It can be explained by the fact that the hyperboloid is tangent to the null cone and therefore, the extension of PV addition to the the null cone is not possible by the formal substitutions above. Surprisingly, by Proposition 5.2 we obtained the induced PV addition on the sphere which is given by the fractional linear mappings $T_{a}(\xi)$.

Formula (38) converges in the limit to the well-known formula in the Euclidean case

$$
\mathrm{e}^{\mathrm{i}\langle-a+x, \lambda \xi\rangle}=\mathrm{e}^{\mathrm{i}\langle-a, \lambda \xi\rangle} e^{\mathrm{i}\langle x, \lambda \xi\rangle}, \quad a, x, \lambda \xi \in \mathbb{R}^{n} .
$$

Proposition 5.3. The function $e_{\lambda, \xi ; t}$ is an eigenfunction of $\Delta_{z, t}$ with eigenvalue $-\lambda^{2}-\frac{(n-1)^{2}}{4 t^{2}}+\frac{n z}{t^{2}}$. 
Proof. Applying $\Delta_{z, t}$ to (38) as a function of $y$ and using Proposition 3.5 we get

$$
\tau_{-x}\left(\Delta_{z, t} e_{\lambda, \xi ; t}\right)(y)=\Delta_{z, t}\left(\tau_{-x} e_{\lambda, \xi ; t}\right)(y)=e_{\lambda, \xi ; t}(x) \Delta_{z, t} e_{\lambda, T_{-x}(\xi) ; t}(y) j_{-x}(0) .
$$

Putting $y=0$ we have

$$
\Delta_{z, t} e_{\lambda, \xi ; t}(x) j_{-x}(0)=\left(\Delta_{z, t} e_{\lambda, T_{-x}(\xi) ; t}\right)(0) e_{\lambda, \xi ; t}(x) j_{-x}(0) .
$$

Thus, we conclude that $e_{\lambda, \xi ; t}(x)$ is an eigenfunction of $\Delta_{z, t}$ with eigenvalue $\Delta_{z, t} e_{\lambda, T_{-x}(\xi) ; t}(0)$. Computing this value we find that the eigenvalue of $e_{\lambda, \xi ; t}(x)$ is $-\lambda^{2}-\frac{(n-1)^{2}}{4 t^{2}}+\frac{n z}{t^{2}}$.

In the limit $t \rightarrow+\infty$ the eigenvalues of $\Delta_{z, t}$ reduce to the eigenvalues of $\Delta$ in $\mathbb{R}^{n}$. In the Euclidean case given two eigenfunctions $\mathrm{e}^{\mathrm{i}\langle x, \lambda \xi\rangle}$ and $\mathrm{e}^{\mathrm{i}\langle x, \gamma \omega\rangle}, \lambda, \gamma \in \mathbb{R}, \xi, \omega \in \mathbb{S}^{n-1}$ of the Laplace operator with eigenvalues $-\lambda^{2}$ and $-\gamma^{2}$ respectively, the product of the two eigenfunctions is again an eigenfunction of the Laplace operator with eigenvalue $-\left(\lambda^{2}+\right.$ $\left.\gamma^{2}+2 \lambda \gamma\langle\xi, \omega\rangle\right)$. Indeed,

$$
\Delta\left(\mathrm{e}^{\mathrm{i}\langle x, \lambda \xi\rangle} \mathrm{e}^{\mathrm{i}\langle x, \gamma \omega\rangle}\right)=-\|\lambda \xi+\gamma \omega\|^{2} \mathrm{e}^{\mathrm{i}\langle x, \lambda \xi+\gamma \omega\rangle}=-\left(\lambda^{2}+\gamma^{2}+2 \lambda \gamma\langle\xi, \omega\rangle\right) \mathrm{e}^{\mathrm{i}\langle x, \lambda \xi+\gamma \omega\rangle} .
$$

Unfortunately, in the hyperbolic case this is no longer true in general. The only exception is the case $n=1$ and $z=0$ as the next proposition shows.

Proposition 5.4. For $n \geq 2$ the product of two eigenfunctions of $\Delta_{z, t}$ is not an eigenfunction of $\Delta_{z, t}$ and for $n=1$ the product of two eigenfunctions of $\Delta_{z, t}$ is an eigenfunction of $\Delta_{z, t}$ only in the case $z=0$.

Proof. Let $e_{\lambda, \xi ; t}$ and $e_{\gamma, \omega ; t}$ be two eigenfunctions of $\Delta_{z, t}$ with eigenvalues $-\lambda^{2}-\frac{(n-1)^{2}}{4 t^{2}}+\frac{n z}{t^{2}}$ and $-\gamma^{2}-\frac{(n-1)^{2}}{4 t^{2}}+\frac{n z}{t^{2}}$ respectively. Since for $n \geq 1$ and $f, g \in C^{2}\left(\mathbb{R}^{n}\right)$

$$
\Delta_{z, t}(f g)=\left(\Delta_{z, t} f\right) g+f\left(\Delta_{z, t} g\right)+2\langle\nabla f, \nabla g\rangle+\frac{2}{t^{2}}\langle x, \nabla f\rangle\langle x, \nabla g\rangle-\frac{z(z+1)}{t^{2}}\left(1-\beta_{x}^{2}\right) f g
$$

we obtain after straightforward computations

$$
\begin{aligned}
& \Delta_{z, t}\left(e_{\lambda, \xi ; t}(x) e_{\gamma, \omega ; t}(x)\right)=\left[-\lambda^{2}-\gamma^{2}-\frac{(n-1)^{2}}{2 t^{2}}+\frac{2 n z}{t^{2}}+\left(\frac{(n-1+2 \mathrm{i} \lambda t)(n-1+2 \mathrm{i} \gamma t)}{t^{2}} \times\right.\right. \\
& \left.\left(\frac{1}{\beta_{x}}-\frac{\langle x, \xi\rangle}{t}\right)^{-1}\left(\frac{1}{\beta_{x}}-\frac{\langle x, \omega\rangle}{t}\right)^{-1}\left(\frac{\|x\|^{2}}{t^{2}}-\frac{\langle x, \omega\rangle}{t \beta_{x}}-\frac{\langle x, \xi\rangle}{t \beta_{x}}+\langle\xi, \omega\rangle+\frac{\langle x, \omega\rangle\langle x, \xi\rangle}{t^{2}}\right)\right) \\
& -\frac{z}{t^{2}}\left(\frac{n-1}{2}+\mathrm{i} \lambda t\right)\left(\frac{1}{\beta_{x}}-\frac{\langle x, \xi\rangle}{t}\right)^{-1}\left(\frac{\|x\|^{2}}{t^{2}} \beta_{x}-\frac{\langle x, \xi\rangle}{t^{2}}\right) \\
& \left.-\frac{z}{t^{2}}\left(\frac{n-1}{2}+\mathrm{i} \gamma t\right)\left(\frac{1}{\beta_{x}}-\frac{\langle x, \omega\rangle}{t}\right)^{-1}\left(\frac{\|x\|^{2}}{t^{2}} \beta_{x}-\frac{\langle x, \omega\rangle}{t^{2}}\right)-\frac{z}{t^{2}}\left(1-\beta_{x}^{2}\right)\right] e_{\lambda, \xi ; t}(x) e_{\gamma, \omega ; t}(x) .
\end{aligned}
$$

Therefore, for $n \geq 2$ and $z \in \mathbb{C}$, the product of two eigenfunctions of $\Delta_{z, t}$ is not an eigenfunction of $\Delta_{z, t}$. For $n=1$ the previous formula reduces to

$$
\begin{aligned}
\Delta_{z, t}\left(e_{\lambda, \xi, t}(x) e_{\gamma, \omega ; t}(x)\right)= & {\left[-\lambda^{2}-\gamma^{2}-2 \lambda \gamma+\frac{2 z}{t^{2}}-\mathrm{i} \lambda t z\left(\frac{1}{\beta_{x}}-\frac{x \xi}{t}\right)^{-1}\left(\frac{x^{2}}{t^{4} \beta_{x}}-\frac{x \xi}{t^{3}}\right)\right.} \\
& \left.-\mathrm{i} \gamma t z\left(\frac{1}{\beta_{x}}-\frac{x \omega}{t}\right)^{-1}\left(\frac{x^{2}}{t^{4} \beta_{x}}-\frac{x \omega}{t^{3}}\right)-\frac{z}{t^{2}}\left(1-\beta_{x}^{2}\right)\right] .
\end{aligned}
$$

For $z=0$, we obtain further

$$
\Delta_{z, t}\left(e_{\lambda, \xi ; t}(x) e_{\gamma, \omega ; t}(x)\right)=-\left(\lambda^{2}+\gamma^{2}+2 \lambda \gamma \xi \omega\right) e_{\lambda, \xi ; t}(x) e_{\gamma, \omega ; t}(x) .
$$

Therefore, only in the case $n=1$ and $z=0$ the product of two eigenfunctions of $\Delta_{z, t}$ is an eigenfunction of $\Delta_{z, t}$. 
In the case when $n=1$ and $z=0$ the hyperbolic plane waves (36) are independent of $\xi$ (we can consider $\xi=1$ ) reducing to

$$
e_{\lambda ; t}(x)=\left(\sqrt{1+\frac{x^{2}}{t^{2}}}-\frac{x}{t}\right)^{-\mathrm{i} \lambda t}
$$

and, therefore, the exponential law is valid, i.e., $e_{\lambda ; t}(x) e_{\gamma ; t}(x)=e_{\lambda+\gamma ; t}(x)$. This explains the special case in Proposition 5.4.

The radial eigenfunctions of $\Delta_{z, t}$ are called generalized spherical functions and assume a very important role in the theory.

Definition 5.5. For each $\lambda \in \mathbb{C}$, we define the generalized spherical function $\phi_{\lambda ; t}$ by

$$
\phi_{\lambda ; t}(x)=\int_{\mathbb{S}^{n-1}} e_{\lambda, \xi ; t}(x) d \sigma(\xi), \quad x \in \mathbb{R}^{n} .
$$

Using (63) and then (65) in Appendix A we can write this function as

$$
\begin{aligned}
\phi_{\lambda ; t}(x) & =\left(1+\frac{\|x\|^{2}}{t^{2}}\right){ }_{2}^{\frac{2 z-n+1-2 \mathrm{i} \lambda t}{4}} F_{1}\left(\frac{n-1+2 \mathrm{i} \lambda t}{4}, \frac{n+1+2 \mathrm{i} \lambda t}{4} ; \frac{n}{2} ; 1-\beta_{x}^{2}\right) \\
& =\left(1+\frac{\|x\|^{2}}{t^{2}}\right){ }_{2}^{\frac{2 z-n+1+2 \mathrm{i} \lambda t}{4}} F_{1}\left(\frac{n-1-2 \mathrm{i} \lambda t}{4}, \frac{n+1-2 \mathrm{i} \lambda t}{4} ; \frac{n}{2} ; 1-\beta_{x}^{2}\right) .
\end{aligned}
$$

Therefore, $\phi_{\lambda ; t}$ is a radial function that satisfies $\phi_{\lambda ; t}=\phi_{-\lambda ; t}$ i.e., $\phi_{\lambda ; t}$ is an even function of $\lambda \in \mathbb{C}$. Applying (66) in Appendix A we obtain further that

$$
\phi_{\lambda ; t}(x)=\left(1+\frac{\|x\|^{2}}{t^{2}}\right)^{\frac{z}{2}}{ }_{2} F_{1}\left(\frac{n-1-2 \mathrm{i} \lambda t}{4}, \frac{n-1+2 \mathrm{i} \lambda t}{4} ; \frac{n}{2} ;-\frac{\|x\|^{2}}{t^{2}}\right) .
$$

Finally, considering $x=t \sinh (s) \xi$, with $s \in \mathbb{R}^{+}$and $\xi \in S^{n-1}$ we have the following relation between $\phi_{\lambda ; t}$ and the Jacobi functions $\varphi_{\lambda t}$ (see (69) in Appendix B):

$$
\phi_{\lambda ; t}(t \sinh (s) \xi)=(\cosh s)^{z} \varphi_{\lambda t}^{\left(\frac{n}{2}-1,-\frac{1}{2}\right)}(s) .
$$

The following theorem characterizes all generalized spherical functions.

Theorem 5.6. The function $\phi_{\lambda ; t}$ is a generalized spherical function with eigenvalue $-\lambda^{2}-$ $\frac{(n-1)^{2}}{4 t^{2}}+\frac{n z}{t^{2}}$. Moreover, if we normalize spherical functions $\phi_{\lambda ; t}$ such that $\phi_{\lambda ; t}(0)=1$, then all generalized spherical functions are given by $\phi_{\lambda ; t}$.

Proof. By Proposition 5.3 it is easy to see that $\phi_{\lambda ; t}$ is an eigenfunction of $\Delta_{z, t}$ with eigenvalue $-\lambda^{2}-\frac{(n-1)^{2}}{4 t^{2}}+\frac{n z}{t^{2}}$. Moreover, $\phi_{\lambda ; t}(0)=1$. Now let $f$ be a spherical function with eigenvalue $-\lambda^{2}-\frac{(n-1)^{2}}{4 t^{2}}+\frac{n z}{t^{2}}$ and consider

$$
f(x)=\left(1+\frac{\|x\|^{2}}{t^{2}}\right)^{\frac{z}{2}} F\left(-\frac{\|x\|^{2}}{t^{2}}\right)
$$

with $F$ a function defined on $\mathbb{R}^{n}$. Since $f$ is a radial function of the form $f(x)=f_{0}(\|x\|)$ then the operator $\Delta_{z, t}$ can be written as

$$
\begin{aligned}
\left(\Delta_{z, t} f\right)(x)= & \left(1+\frac{\|x\|^{2}}{t^{2}}\right) f_{0}^{\prime \prime}(\|x\|)+f_{0}^{\prime}(\|x\|)\left(\frac{n-1}{\|x\|}+(n-2 z) \frac{\|x\|}{t^{2}}\right) \\
& +\frac{z(z+1)}{t^{2}}\left(1-\beta_{x}^{2}\right) f_{0}(\|x\|) .
\end{aligned}
$$


Then, considering $\|x\|^{2}=r^{2}$ and after straightforward computations we see that if $f$ given by (43) is an eigenfunction of $\Delta_{z, t}$ then $F$ satisfies the following hypergeometric equation:

$$
\begin{aligned}
\left(-\frac{r^{2}}{t^{2}}\right)\left(1-\left(-\frac{r^{2}}{t^{2}}\right)\right) & F^{\prime \prime}\left(-\frac{r^{2}}{t^{2}}\right)+\left(\frac{n}{2}-\left(\frac{n+1}{2}\right)\left(-\frac{r^{2}}{t^{2}}\right)\right) F^{\prime}\left(-\frac{r^{2}}{t^{2}}\right) \\
& -\left(\frac{n-1-2 \mathrm{i} \lambda t}{4}\right)\left(\frac{n+1+2 \mathrm{i} \lambda t}{4}\right) F\left(-\frac{r^{2}}{t^{2}}\right)=0 .
\end{aligned}
$$

The smooth solutions at 0 of the last equation are multiples of ${ }_{2} F_{1}\left(\frac{n-1-2 \mathrm{i} \lambda t}{4}, \frac{n-1+2 \mathrm{i} \lambda t}{4} ; \frac{n}{2} ;-\frac{\|x\|^{2}}{t^{2}}\right)$. Therefore, by (41) $f$ is a constant multiple of $\phi_{\lambda ; t}$.

Now we study the asymptotic behavior of $\phi_{\lambda ; t}$ at infinity to obtain the Harish-Chandra $c$-function in our case.

Lemma 5.7. For $\operatorname{Im}(\lambda)<0$ we have

$$
\lim _{s \rightarrow+\infty} \phi_{\lambda ; t}(t \sinh s) e^{\left(\frac{n-1}{2}-z-\mathrm{i} \lambda t\right) s}=c(\lambda t)
$$

where $c(\lambda t)$ is the Harish-Chandra c-function given by

$$
c(\lambda t)=\frac{2^{n-2-z}}{\sqrt{\pi}} \frac{\Gamma\left(\frac{n}{2}\right) \Gamma(\mathrm{i} \lambda t)}{\Gamma\left(\frac{n-1}{2}+\mathrm{i} \lambda t\right)} .
$$

Proof. Considering (42), (72) and (71) in Appendix B, and the limit

$$
\lim _{s \rightarrow+\infty} e^{s} / \cosh (s)=2
$$

we obtain

$$
\begin{aligned}
\lim _{s \rightarrow+\infty} \phi_{\lambda ; t}(t \sinh s) e^{\left(\frac{n-1}{2}-z-i \lambda t\right) s} & =\lim _{s \rightarrow \infty} e^{-z s}(\cosh s)^{z} \varphi_{\lambda t}^{\left(\frac{n}{2}-1,-\frac{1}{2}\right)}(s) e^{\left(-\mathrm{i} \lambda t+\frac{n-1}{2}\right) s} \\
& =2^{-z} c_{\frac{n}{2}-1,-\frac{1}{2}}(\lambda t) \\
& =\frac{2^{\frac{n-1}{2}-z-\mathrm{i} \lambda t} \Gamma\left(\frac{n}{2}\right) \Gamma(\mathrm{i} \lambda t)}{\Gamma\left(\frac{n-1+2 \mathrm{i} \lambda t}{4}\right) \Gamma\left(\frac{n+1+2 \mathrm{i} \lambda t}{4}\right)}
\end{aligned}
$$

Using the relation $\Gamma(z) \Gamma\left(z+\frac{1}{2}\right)=2^{1-2 z} \sqrt{\pi} \Gamma(2 z)$ we can write

$$
\Gamma\left(\frac{n+1+2 \mathrm{i} \lambda t}{4}\right)=\Gamma\left(\frac{n-1+2 \mathrm{i} \lambda t}{4}+\frac{1}{2}\right)=\frac{2^{1-\frac{n-1+2 \mathrm{i} \lambda t}{2}} \sqrt{\pi} \Gamma\left(\frac{n-1+2 \mathrm{i} \lambda t}{2}\right)}{\Gamma\left(\frac{n-1+2 \mathrm{i} \lambda t}{4}\right)}
$$

and, therefore, (45) simplifies to

$$
c(\lambda t)=\frac{2^{n-2-z}}{\sqrt{\pi}} \frac{\Gamma\left(\frac{n}{2}\right) \Gamma(\mathrm{i} \lambda t)}{\Gamma\left(\frac{n-1}{2}+\mathrm{i} \lambda t\right)} .
$$

Finally, we prove the addition formula for spherical functions.

Proposition 5.8. For every $\lambda \in \mathbb{C}, t \in \mathbb{R}^{+}$, and $a, x \in \mathbb{R}^{n}$

$$
\begin{aligned}
\tau_{a} \phi_{\lambda ; t}(x) & =j_{a}(0) \int_{\mathbb{S}^{n-1}} e_{-\lambda, \xi ; t}(a) e_{\lambda, \xi ; t}(x) d \sigma(\xi) \\
& =j_{a}(0) \int_{\mathbb{S}^{n-1}} e_{\lambda, \xi ; t}(a) e_{-\lambda, \xi ; t}(x) d \sigma(\xi) .
\end{aligned}
$$


Proof. By (38) we have

$$
\begin{aligned}
\tau_{a} \phi_{\lambda ; t}(x) & =\int_{\mathbb{S}^{n-1}} \tau_{a} e_{\lambda, \xi ; t}(x) d \sigma(\xi) \\
& =j_{a}(0) \int_{\mathbb{S}^{n-1}} e_{\lambda, \xi ; t}(-a) e_{\lambda, T_{a}(\xi) ; t}(x) d \sigma(\xi) .
\end{aligned}
$$

Making the change of variables $T_{a}(\xi)=\xi^{\prime} \Leftrightarrow \xi=T_{-a}\left(\xi^{\prime}\right)$ the measure becomes

$$
d \sigma(\xi)=\left(\frac{1}{\frac{1}{\beta_{a}}-\frac{\left\langle a, \xi^{\prime}\right\rangle}{t}}\right)^{n-1} d \sigma\left(\xi^{\prime}\right)
$$

Thus,

$$
\tau_{a} \phi_{\lambda ; t}(x)=j_{a}(0) \int_{\mathbb{S}^{n-1}} e_{\lambda, T_{-a}\left(\xi^{\prime}\right) ; t}(-a) e_{\lambda, \xi^{\prime} ; t}(x)\left(\frac{1}{\frac{1}{\beta_{a}}-\frac{\left\langle a, \xi^{\prime}\right\rangle}{t}}\right)^{n-1} d \sigma\left(\xi^{\prime}\right) .
$$

From (36) and (39) we obtain

$$
e_{\lambda, T_{-a}\left(\xi^{\prime}\right) ; t}(-a)=\frac{\left(\beta_{a}\right)^{-z+\frac{n-1}{2}+\mathrm{i} \lambda t}}{\left(\beta_{a}\right)^{n-1+2 \mathrm{i} \lambda t}\left(1-\frac{\left\langle\beta_{a} a, \xi^{\prime}\right\rangle}{t}\right)^{-\frac{n-1}{2}-\mathrm{i} \lambda t}} .
$$

Since

$$
\begin{aligned}
e_{\lambda, T_{-a}\left(\xi^{\prime}\right) ; t}(-a)\left(\frac{1}{\frac{1}{\beta_{a}}-\frac{\left\langle a, \xi^{\prime}\right\rangle}{t}}\right)^{n-1}= & \frac{\left(\beta_{a}\right)^{-z+\frac{n-1}{2}+\mathrm{i} \lambda t}}{\left(\beta_{a}\right)^{n-1+2 \mathrm{i} \lambda t}\left(1-\frac{\left\langle\beta_{a} a, \xi^{\prime}\right\rangle}{t}\right)^{-\frac{n-1}{2}-\mathrm{i} \lambda t}} \\
& \times \frac{\left(\beta_{a}\right)^{n-1}}{\left(1-\frac{\left\langle\beta_{a} a, \xi^{\prime}\right\rangle}{t}\right)^{n-1}} \\
= & \frac{\left(\beta_{a}\right)^{-z+\frac{n-1}{2}-\mathrm{i} \lambda t}}{\left(1-\frac{\left\langle\beta_{a} a, \xi^{\prime}\right\rangle}{t}\right)^{\frac{n-1}{2}-\mathrm{i} \lambda t}} \\
= & e_{-\lambda, \xi^{\prime} ; t}(a)
\end{aligned}
$$

we finally obtain

$$
\tau_{a} \phi_{\lambda ; t}(x)=j_{a}(0) \int_{\mathbb{S}^{n-1}} e_{-\lambda, \xi^{\prime} ; t}(a) e_{\lambda, \xi^{\prime} ; t}(x) d \sigma\left(\xi^{\prime}\right)
$$

The second equality follows from the fact that $\phi_{\lambda ; t}$ is an even function of $\lambda$, i.e., $\phi_{\lambda ; t}=\phi_{-\lambda ; t}$.

\section{The generalized Poisson transform}

Definition 6.1. Let $f \in L^{2}\left(\mathbb{S}^{n-1}\right)$. Then the generalized Poisson transform is defined by

$$
P_{\lambda, t} f(x)=\int_{\mathbb{S}^{n-1}} e_{\lambda, \xi ; t}(x) f(\xi) d \sigma(\xi), \quad x \in \mathbb{R}^{n} .
$$


For a spherical harmonic $Y_{k}$ of degree $k$ and applying (62) in Appendix A we obtain:

$$
\begin{aligned}
\left(P_{\lambda, t} Y_{k}\right)(x)= & C_{k, \nu}\left(\beta_{x}\right)^{-z+\frac{n-1}{2}+i \lambda t} \times \\
& { }_{2} F_{1}\left(\frac{\nu+k}{2}, \frac{\nu+k+1}{2} ; k+\frac{n}{2} ; 1-\beta_{x}^{2}\right) Y_{k}\left(\beta_{x} \frac{x}{t}\right)
\end{aligned}
$$

with $\nu=\frac{n-1}{2}+\mathrm{i} \lambda t$, and $C_{k, \nu}=2^{-k} \frac{(\nu)_{k}}{(n / 2)_{k}}$. For $f=\sum_{k=0}^{\infty} a_{k} Y_{k} \in L^{2}\left(\mathbb{S}^{n-1}\right)$ then we have

$$
P_{\lambda, t} f(x)=\sum_{k=0}^{\infty} a_{k} C_{k, \nu}\left(\beta_{x}\right)^{-z+\frac{n-1}{2}+i \lambda t}{ }_{2} F_{1}\left(\frac{\nu+k}{2}, \frac{\nu+k+1}{2} ; k+\frac{n}{2} ; 1-\beta_{x}^{2}\right) Y_{k}\left(\beta_{x} \frac{x}{t}\right) .
$$

Now we prove a result about the injectivity of the generalized Poisson transform which is important later on.

Proposition 6.2. The Poisson transform $P_{\lambda, t}$ is injective in $L^{2}\left(\mathbb{S}^{n-1}\right)$ if and only if $\lambda \neq \mathrm{i}\left(\frac{2 k+n-1}{2 t}\right)$ for all $k \in \mathbb{Z}^{+}$.

Proof. Let $\lambda=\mathrm{i}\left(\frac{2 k_{0}+n-1}{2 t}\right)$ for some $k_{0} \in \mathbb{Z}^{+}$. Then by (47) we have that $P_{\lambda, t} Y_{k}=0$, for all $k>k_{0}$ since $((n-1) / 2+\mathrm{i} \lambda t)_{k}$ is zero. Conversely, if $\lambda \neq \mathrm{i}\left(\frac{2 k+n-1}{2 t}\right)$ for all $k \in \mathbb{Z}^{+}$, then all the coefficients $((n-1) / 2+\mathrm{i} \lambda t)_{k}$ are not vanishing for $k \in \mathbb{Z}^{+}$. Hence, by (47) we have that $P_{\lambda, t} f=0$ if and only if $f=0$. Thus, $P_{\lambda, t}$ is injective for every $\lambda \neq \mathrm{i}\left(\frac{2 k+n-1}{2 t}\right)$, $k \in \mathbb{Z}^{+}$.

Corollary 6.3. Let $\lambda \neq \mathrm{i}\left(\frac{2 k+n-1}{2 t}\right), k \in \mathbb{Z}^{+}$. Then for $f$ in $C_{0}^{\infty}\left(\mathbb{R}^{n}\right)$ the space of functions $\widehat{f}(\lambda, \xi)$ is dense in $L^{2}\left(\mathbb{S}^{n-1}\right)$.

Proof. Let $g \in L^{2}\left(\mathbb{S}^{n-1}\right)$ be such that

$$
\int_{\mathbb{S}^{n-1}} g(\xi) \widehat{f}(\lambda, \xi) d \sigma(\xi)=0
$$

for all $f \in C_{0}^{\infty}\left(\mathbb{R}^{n}\right)$. Therefore,

$$
\int_{\mathbb{R}^{n}} f(x)\left(\int_{\mathbb{S}^{n-1}} g(\xi) e_{-\lambda, \xi ; t}(x) d \sigma(\xi)\right) d \mu_{z, t}(x)=0
$$

for all $f \in C_{0}^{\infty}\left(\mathbb{R}^{n}\right)$, which implies that for every $x \in \mathbb{R}^{n}$

$$
P_{-\lambda, t} g(x)=\int_{\mathbb{S}^{n-1}} g(\xi) e_{-\lambda, \xi ; t}(x) d \sigma(\xi)=0
$$

Finally, by Proposition 6.2 we have $g=0$.

\section{The generalized Helgason Fourier transform}

Definition 7.1. For $f \in C_{0}^{\infty}\left(\mathbb{R}^{n}\right), \lambda \in \mathbb{C}$ and $\xi \in \mathbb{S}^{n-1}$ we define the generalized Helgason Fourier transform of $f$ as

$$
\widehat{f}(\lambda, \xi ; t)=\int_{\mathbb{R}^{n}} e_{-\lambda, \xi ; t}(x) f(x) d \mu_{z, t}(x) .
$$


Remark 3. If $f$ is a radial function i.e., $f(x)=f(\|x\|)$, then $\widehat{f}(\lambda, \xi ; t)$ is independent of $\xi$ and reduces by (40) to the spherical transform of $f$ defined by

$$
\widehat{f}(\lambda ; t)=\int_{\mathbb{R}^{n}} \phi_{-\lambda ; t}(x) f(x) d \mu_{z, t}(x) .
$$

Moreover, by (37) we recover in the Euclidean limit the usual Fourier transform in $\mathbb{R}^{n}$.

From Propositions 3.8 and 5.3 we obtain the following result.

Proposition 7.2. If $f \in C_{0}^{\infty}\left(\mathbb{R}^{n}\right)$ then

$$
\widehat{\Delta_{z, t} f}(\lambda, \xi ; t)=-\left(\lambda^{2}+\frac{(n-1)^{2}}{4 t^{2}}-\frac{n z}{t^{2}}\right) \widehat{f}(\lambda, \xi ; t) .
$$

Now we study the hyperbolic convolution theorem with respect to the generalized Helgason Fourier transform. We begin with the following lemma.

Lemma 7.3. For $a \in \mathbb{R}^{n}$ and $f \in C_{0}^{\infty}\left(\mathbb{R}^{n}\right)$

$$
\widehat{\tau_{a} f}(\lambda, \xi ; t)=j_{a}(0) e_{-\lambda, \xi ; t}(a) \widehat{f}\left(\lambda, T_{-a}(\xi) ; t\right) .
$$

Proof. By (31) and (38) we have

$$
\begin{aligned}
\widehat{\tau_{a} f}(\lambda, \xi ; t) & =\int_{\mathbb{R}^{n}} e_{-\lambda, \xi ; t}(x) \tau_{a} f(x) d \mu_{z, t}(x) \\
& =\int_{\mathbb{R}^{n}} \tau_{-a} e_{-\lambda, \xi ; t}(x) f(x) d \mu_{z, t}(x) \\
& =j_{a}(0) e_{-\lambda, \xi ; t}(a) \int_{\mathbb{R}^{n}} e_{-\lambda, T_{-a}(\xi) ; t}(x) f(x) d \mu_{z, t}(x) \\
& =j_{a}(0) e_{-\lambda, \xi ; t}(a) \widehat{f}\left(\lambda, T_{-a}(\xi) ; t\right) .
\end{aligned}
$$

Theorem 7.4 (Generalized Hyperbolic Convolution Theorem). Let $f, g \in C_{0}^{\infty}\left(\mathbb{R}^{n}\right)$. Then

$$
\widehat{f * g}(\lambda, \xi)=\int_{\mathbb{R}^{n}} f(y) e_{-\lambda, \xi ; t}(y) \widehat{\widetilde{g}_{y}}\left(\lambda, T_{-y}(\xi) ; t\right) d \mu_{z, t}(y)
$$

where $\widetilde{g}_{y}(x)=g(\operatorname{gyr}[y, x] x)$.

Proof. Let $I=\widehat{f * g}(\lambda, \xi)$. We have

$$
\begin{aligned}
I & =\int_{\mathbb{R}^{n}}\left(\int_{\mathbb{R}^{n}} f(y) \tau_{x} g(-y) j_{x}(x) d \mu_{z, t}(y)\right) e_{-\lambda, \xi ; t}(x) d \mu_{z, t}(x) \\
& =\int_{\mathbb{R}^{n}} f(y)\left(\int_{\mathbb{R}^{n}} \tau_{x} g(-y) e_{-\lambda, \xi ; t}(x) j_{x}(x) d \mu_{z, t}(x)\right) d \mu_{z, t}(y) \text { (Fubini) } \\
& =\int_{\mathbb{R}^{n}} f(y)\left(\int_{\mathbb{R}^{n}} \tau_{y} g(\operatorname{gyr}[x, y] x) e_{-\lambda, \xi ; t}(x) j_{y}(y) d \mu_{z, t}(x)\right) d \mu_{z, t}(y) \\
& =\int_{\mathbb{R}^{n}} f(y) \widehat{\tau_{y} \widetilde{g}_{y}}(\lambda, \xi ; t) j_{y}(y) d \mu_{z, t}(y) \\
& =\int_{\mathbb{R}^{n}} f(y) e_{-\lambda, \xi ; t}(y) \widehat{\widehat{g}_{y}}\left(\lambda, T_{-y}(\xi) ; t\right) d \mu_{z, t}(y) \quad(\text { by } \quad(49),(16))
\end{aligned}
$$


Since in the limit $t \rightarrow+\infty$ gyrations reduce to the identity and $(-y) \oplus \xi$ reduces to $\xi$, formula (50) converges in the Euclidean limit to the well-know Convolution Theorem: $\widehat{f * g}=\widehat{f} \cdot \widehat{g}$. By Remark 3 if $g$ is a radial function we obtain the pointwise product of the generalized Helgason Fourier transforms.

Corollary 7.5. Let $f, g \in C_{0}^{\infty}\left(\mathbb{R}^{n}\right)$ and $g$ radial. Then

$$
\widehat{f * g}(\lambda, \xi ; t)=\widehat{f}(\lambda, \xi ; t) \widehat{g}(\lambda ; t) .
$$

\section{Inversion of the generalized Helgason Fourier transform and Plancherel's Theorem}

First we obtain an inversion formula for the radial case, that is, for the generalized spherical transform.

Lemma 8.1. The generalized spherical transform denoted by $\mathcal{H}$ can be written as

$$
\mathcal{H}=\mathcal{J}_{\frac{n}{2}-1,-\frac{1}{2}} \circ M_{z}
$$

where $\mathcal{J}_{\frac{n}{2}-1,-\frac{1}{2}}$ is the Jacobi transform (see (68) in Appendix B) with parameters $\alpha=\frac{n}{2}-1$ and $\beta=-\frac{1}{2}$ and

$$
\left(M_{z, t} f\right)(s):=2^{1-n} A_{n-1} t^{n}(\cosh s)^{-z} f(t \sinh s) .
$$

Proof. Integrating (48) in polar coordinates $x=t \sinh (s) \xi s>0, \xi \in S^{n-1}$ we obtain

$$
\widehat{f}(\lambda ; t)=A_{n-1} \int_{0}^{+\infty} f(t \sinh (s) \xi) \phi_{-\lambda ; t}(t \sinh (s) \xi)(\cosh (s))^{-2 z} t^{n}(\sinh (s))^{n-1} d s
$$

Applying (42) yields

$$
\begin{aligned}
\widehat{f}(\lambda ; t) & =2^{1-n} A_{n-1} t^{n} \int_{0}^{+\infty} f(t \sinh s)(\cosh s)^{-z} \varphi_{\lambda t}^{\left(\frac{n}{2}-1,-\frac{1}{2}\right)}(s)(2 \sinh s)^{n-1} d s \\
& =\left(\mathcal{J}_{\frac{n}{2}-1,-\frac{1}{2}} \circ M_{z, t} f\right)(\lambda t) .
\end{aligned}
$$

Lemma 8.1 allow us to obtain a Paley-Wiener Theorem for the generalized Helgason Fourier transform by using the Paley-Wiener Theorem for the Jacobi transform (Theorem B.1). Let $C_{0, R}^{\infty}\left(\mathbb{R}^{n}\right)$ denotes the space of all radial $C^{\infty}$ functions on $\mathbb{R}^{n}$ with compact support and $\mathcal{E}\left(\mathbb{C} \times S^{n-1}\right)$ the space of functions $g(\lambda, \xi)$ on $\mathbb{C} \times \mathbb{S}^{n-1}$, even and holomorphic in $\lambda$ and of uniform exponential type, i.e., there is a positive constant $A_{g}$ such that for all $n \in \mathbb{N}$

$$
\sup _{(\lambda, \xi) \in \mathbb{C} \times \mathbb{S}^{n-1}}|g(\lambda, \xi)|(1+|\lambda|)^{n} e^{A_{g}|\operatorname{Im}(\lambda)|}<\infty
$$

where $\operatorname{Im}(\lambda)$ denotes the imaginary part of $\lambda$.

Corollary 8.2. (Paley-Wiener Theorem) The generalized Helgason Fourier transform is bijective from $C_{0, R}^{\infty}\left(\mathbb{R}^{n}\right)$ onto $\mathcal{E}\left(\mathcal{C} \times \mathbb{S}^{n-1}\right)$.

From now on we denote $C_{n, t, z}=\frac{1}{2^{2 z-n+2} t^{n-1} \pi A_{n-1}}$. 
Theorem 8.3. For all $f \in C_{0, R}^{\infty}\left(\mathbb{R}^{n}\right)$ we have the inversion formula

$$
f(x)=C_{n, t, z} \int_{0}^{+\infty} \widehat{f}(\lambda ; t) \phi_{\lambda ; t}(x)|c(\lambda t)|^{-2} d \lambda
$$

Proof. Applying formula (70) in Appendix B for the Jacobi transform and Lemma 6.7 we obtain

$$
\begin{aligned}
M_{z, t} f(s) & =\frac{1}{2 \pi} \int_{0}^{+\infty} \widehat{f}(\lambda ; t) \varphi_{\lambda t}^{\left(\frac{n}{2}-1,-\frac{1}{2}\right)}(s)\left|c_{\frac{n}{2}-1,-\frac{1}{2}}(\lambda t)\right|^{-2} t d \lambda \\
& =\frac{1}{2 \pi} \int_{0}^{+\infty} \widehat{f}(\lambda ; t)(\cosh s)^{-z} \phi_{\lambda ; t}(x) \frac{|c(\lambda t)|^{-2}}{2^{2 z}} t d \lambda .
\end{aligned}
$$

In the last equality we use (42) and (45). Applying (51) we obtain

$$
f(t \sinh s)=C_{n, t, z} \int_{0}^{+\infty} \widehat{f}(\lambda ; t) \phi_{\lambda ; t}(x)|c(\lambda t)|^{-2} d \lambda .
$$

Since $f$ is radial and $\|x\|=t \sinh s$ we obtain the desired result.

Remark 4. The inversion formula (52) can be written as

$$
f(x)=\frac{C_{n, t, z}}{2} \int_{\mathbb{R}} \widehat{f}(\lambda ; t) \phi_{\lambda ; t}(x)|c(\lambda t)|^{-2} d \lambda
$$

since the integrand is an even function of $\lambda \in \mathbb{R}$. Note that $f$ is radial and therefore $\widehat{f}(\lambda ; t)$ is an even function of $\lambda, \phi_{\lambda ; t}=\phi_{-\lambda ; t}$, and $|c(-\lambda t)|=|\overline{c(\lambda t)}|=|c(\lambda t)|$, for $\lambda \in \mathbb{R}$.

Now that we have an inversion formula for the radial case we present our main results, the inversion formula for the generalized Helgason Fourier transform and the associated Plancherel's Theorem.

Proposition 8.4. For $f \in C_{0}^{\infty}\left(\mathbb{R}^{n}\right)$ and $\lambda \in \mathbb{C}$ holds

$$
f * \phi_{\lambda ; t}(x)=\int_{\mathbb{S}^{n-1}} \widehat{f}(\lambda, \xi ; t) e_{\lambda, \xi ; t}(x) d \sigma(\xi) .
$$

Proof. By (46), (16), Fubini's Theorem, and the fact that $\phi$ is a radial function we have

$$
\begin{aligned}
f * \phi_{\lambda ; t}(x) & =\int_{\mathbb{R}^{n}} f(y) \tau_{x} \phi_{\lambda ; t}(y) j_{x}(x) d \mu_{z, t}(y) \\
& =\int_{\mathbb{R}^{n}} f(y)\left(\int_{\mathbb{S}^{n-1}} e_{\lambda, \xi ; t}(x) e_{-\lambda, \xi ; t}(y) j_{x}(0) j_{x}(x) d \sigma(\xi)\right) d \mu_{z, t}(y) \\
& =\int_{\mathbb{S}^{n-1}}\left(\int_{\mathbb{R}^{n}} f(y) e_{-\lambda, \xi ; t}(y) d \mu_{z, t}(y)\right) e_{\lambda, \xi ; t}(x) d \sigma(\xi) \\
& =\int_{\mathbb{S}^{n-1}} \widehat{f}(\lambda, \xi ; t) e_{\lambda, \xi ; t}(x) d \sigma(\xi) .
\end{aligned}
$$

Theorem 8.5. (Inversion formula) If $f \in C_{0}^{\infty}\left(\mathbb{R}^{n}\right)$ then

$$
f(x)=C_{n, t, z} \int_{0}^{+\infty} \int_{\mathbb{S}^{n-1}} \widehat{f}(\lambda, \xi ; t) e_{\lambda, \xi ; t}(x)|c(\lambda t)|^{-2} d \sigma(\xi) d \lambda .
$$


Proof. Given $f \in C_{0}^{\infty}\left(B_{n}^{t}\right)$ and $x, y \in \mathbb{R}^{n}$ we consider the radial function

$$
f_{x}(y)=\int_{\mathrm{SO}(n)} \tau_{K^{-1} x} f(-K y) j_{x}(x) d K,
$$

where $K \in \mathrm{SO}(n)$ and $d K$ is the normalised Haar measure on $\mathrm{SO}(n)$. Applying the inversion formula (52) we get

$$
f_{x}(y)=C_{n, t, z} \int_{0}^{+\infty} \widehat{f}_{x}(\lambda ; t) \phi_{\lambda ; t}(y)|c(\lambda t)|^{-2} d \lambda .
$$

By (48) and Fubini's Theorem we have

$$
\begin{aligned}
\widehat{f}_{x}(\lambda ; t) & =\int_{\mathbb{R}^{n}}\left(\int_{\mathrm{SO}(n)} \tau_{K^{-1} x} f(-K y) j_{x}(x) d K\right) \phi_{-\lambda ; t}(y) d \mu_{z, t}(y) \\
& =\int_{\mathrm{SO}(n)}\left(\int_{\mathbb{R}^{n}} f(x \oplus(-K y)) j_{K^{-1} x}(y) j_{x}(x) \phi_{-\lambda ; t}(y) d \mu_{z, t}(y)\right) d K
\end{aligned}
$$

Considering the change of variables $K y \mapsto z$ we see that the inner integral is independent on $K$. Then we obtain

$$
\begin{aligned}
\widehat{f_{x}}(\lambda ; t) & =\int_{\mathbb{R}^{n}} \tau_{x} f(-z) \phi_{-\lambda ; t}(z) j_{x}(x) d \mu_{z, t}(z) \\
& =\left(f * \phi_{\lambda ; t}\right)(x) .
\end{aligned}
$$

Since $f(x)=f_{x}(0)$ it follows from (56), (57), and (54) that

$$
\begin{aligned}
f(x) & =C_{n, t, z} \int_{0}^{+\infty} \widehat{f}_{x}(\lambda ; t) \phi_{\lambda ; t}(0)|c(\lambda t)|^{-2} d \lambda \\
& =C_{n, t, z} \int_{0}^{+\infty}\left(f * \phi_{\lambda ; t}\right)(x)|c(\lambda t)|^{-2} d \lambda \\
& =C_{n, t, z} \int_{0}^{+\infty} \int_{\mathbb{S}^{n-1}} \widehat{f}(\lambda, \xi ; t) e_{\lambda, \xi ; t}(x)|c(\lambda t)|^{-2} d \sigma(\xi) d \lambda .
\end{aligned}
$$

Remark 5. Applying the inversion formula (53) in the proof of Theorem 8.5 we can write the inversion formula (55) as

$$
f(x)=\frac{C_{n, t, z}}{2} \int_{\mathbb{R}} \int_{\mathbb{S}^{n-1}} \widehat{f}(\lambda, \xi ; t) e_{\lambda, \xi ; t}(x)|c(\lambda t)|^{-2} d \sigma(\xi) d \lambda .
$$

\section{Theorem 8.6. (Plancherel's Theorem)}

The generalized Helgason Fourier transform extends to an isometry from $L^{2}\left(\mathbb{R}^{n}, d \mu_{z, t}\right)$ onto $L^{2}\left(\mathbb{R}^{+} \times \mathbb{S}^{n-1}, C_{n, t, z}|c(\lambda t)|^{-2} d \lambda d \sigma\right)$, i.e.,

$$
\int_{\mathbb{R}^{n}}|f(x)|^{2} d \mu_{z, t}(x)=C_{n, t, z} \int_{0}^{+\infty} \int_{\mathbb{S}^{n-1}}|\widehat{f}(\lambda, \xi ; t)|^{2}|c(\lambda t)|^{-2} d \sigma(\xi) d \lambda .
$$


Proof. For $f, g \in C_{0}^{\infty}\left(\mathbb{R}^{n}\right)$ we obtain Parseval's relation by the inversion formula (55) and Fubini's Theorem:

$$
\begin{aligned}
& C_{n, t, z} \int_{0}^{+\infty} \int_{S^{n-1}} \widehat{f}(\lambda, \xi ; t) \overline{\widehat{g}(\lambda, \xi ; t)}|c(\lambda t)|^{-2} d \sigma(\xi) d \lambda \\
& =C_{n, t, z} \int_{0}^{+\infty} \int_{S^{n-1}} \widehat{f}(\lambda, \xi ; t) \int_{\mathbb{R}^{n}} \overline{g(x)} e_{\lambda, \xi ; t}(x) d \mu_{z, t}(x)|c(\lambda t)|^{-2} d \sigma(\xi) d \lambda \\
& =\int_{\mathbb{R}^{n}}\left[C_{n, t, z} \int_{0}^{+\infty} \int_{S^{n-1}} \widehat{f}(\lambda, \xi ; t) e_{\lambda, \xi ; t}(x)|c(\lambda t)|^{-2} d \sigma(\xi) d \lambda\right] \overline{g(x)} d \mu_{z, t}(x) \\
& =\int_{\mathbb{R}^{n}} f(x) \overline{g(x)} d \mu_{z, t}(x) .
\end{aligned}
$$

By taking $f=g$ we obtain (58) for $f \in C_{0}^{\infty}\left(\mathbb{R}^{n}\right)$ and the result can be extended to $L^{2}\left(\mathbb{R}^{n}, d \mu_{z, t}\right)$ since $C_{0}^{\infty}\left(\mathbb{R}^{n}\right)$ is dense in $L^{2}\left(\mathbb{R}^{n}, d \mu_{z, t}\right)$. It remains to prove the surjectivity of the generalized Helgason Fourier transform. This can be done in a similar way as in ([15], Theorem 6.14) and therefore we omit the details.

Having obtained the main results we now study the limit $t \rightarrow+\infty$ of the previous results. It is anticipated that in the Euclidean limit we recover the usual inversion formula for the Fourier transform and Plancherel's Theorem on $\mathbb{R}^{n}$. To see that this is indeed the case, we observe that from (44)

$$
\frac{1}{|c(\lambda t)|^{2}}=\frac{\left(A_{n-1}\right)^{2}}{\pi^{n-1} 2^{2 n-2 z-2}}\left|\frac{\Gamma\left(\frac{n-1}{2}+\mathrm{i} \lambda t\right)}{\Gamma(\mathrm{i} \lambda t)}\right|^{2},
$$

with $A_{n-1}=\frac{2 \pi^{\frac{n}{2}}}{\Gamma\left(\frac{n}{2}\right)}$ being the surface area of $\mathbb{S}^{n-1}$. Finally, using (59) the generalized Helgason inverse Fourier transform (55) simplifies to

$$
\begin{aligned}
f(x) & =\frac{A_{n-1}}{(2 \pi)^{n} t^{n-1}} \int_{0}^{+\infty} \int_{\mathbb{S}^{n-1}} \widehat{f}(\lambda, \xi ; t) e_{\lambda, \xi ; t}(x)\left|\frac{\Gamma\left(\frac{n-1}{2}+\mathrm{i} \lambda t\right)}{\Gamma(\mathrm{i} \lambda t)}\right|^{2} d \sigma(\xi) d \lambda \\
& =\frac{1}{(2 \pi)^{n}} \int_{0}^{+\infty} \int_{\mathbb{S}^{n-1}} \widehat{f}(\lambda, \xi ; t) e_{\lambda, \xi ; t}(x) \frac{\lambda^{n-1}}{N^{(n)}(\lambda t)} d \xi d \lambda
\end{aligned}
$$

with

$$
N^{(n)}(\lambda t)=\left|\frac{\Gamma(i \lambda t)}{\Gamma\left(\frac{n-1}{2}+\mathrm{i} \lambda t\right)}\right|^{2}(\lambda t)^{n-1} .
$$

Some particular values are $N^{(1)}(\lambda t)=1, N^{(2)}(\lambda t)=\operatorname{coth}(\lambda t), N^{(3)}=1$, and $N^{(4)}(\lambda t)=$ $\frac{(2 \lambda t)^{2} \operatorname{coth}(\pi \lambda t)}{1+(2 \lambda t)^{2}}$. Since $\lim _{t \rightarrow+\infty} N^{(n)}(\lambda t)=1$, for any $n \in \mathbb{N}$ and $\lambda \in \mathbb{R}^{+}$(see [1]), we conclude that in the Euclidean limit the generalized Helgason inverse Fourier transform (60) converges to the usual inverse Fourier transform in $\mathbb{R}^{n}$ written in polar coordinates:

$$
f(x)=\frac{1}{(2 \pi)^{n}} \int_{0}^{+\infty} \int_{\mathbb{S}^{n-1}} \widehat{f}(\lambda \xi) \mathrm{e}^{\mathrm{i}\langle x, \lambda \xi\rangle} \lambda^{n-1} d \xi d \lambda, \quad x, \lambda \xi \in \mathbb{R}^{n} .
$$

Finally, Plancherel's Theorem (58) can be written as

$$
\int_{\mathbb{R}^{n}}|f(x)|^{2} d \mu_{z, t}(x)=\frac{1}{(2 \pi)^{n}} \int_{0}^{+\infty} \int_{\mathbb{S}^{n-1}}|\widehat{f}(\lambda, \xi)|^{2} \frac{\lambda^{n-1}}{N^{(n)}(\lambda t)} d \xi d \lambda
$$


and, therefore, we have an isometry between the spaces $L^{2}\left(\mathbb{R}^{n}, d \mu_{z, t}\right)$ and $L^{2}\left(\mathbb{R}^{+} \times \mathbb{S}^{n-1}, \frac{\lambda^{n-1}}{(2 \pi)^{n} N^{(n)}(\lambda t)} d \lambda d \xi\right)$. Applying the limit $t \rightarrow+\infty$ to (61) we recover Plancherel's Theorem in $\mathbb{R}^{n}$ :

$$
\int_{\mathbb{R}^{n}}|f(x)|^{2} d x=\frac{1}{(2 \pi)^{n}} \int_{0}^{+\infty} \int_{\mathbb{S}^{n-1}}|\widehat{f}(\lambda \xi)|^{2} \lambda^{n-1} d \xi d \lambda
$$

\section{Heat kernel associated to the proper velocity gyrogroup}

We consider the following initial value problem for the heat equation associated to the generalized Laplace Beltrami operator $\Delta_{z, t}$ :

$$
\left\{\begin{array}{l}
\partial_{\tau} u(x, \tau)=\Delta_{z, t} u(x, \tau) \\
u(x, 0)=f(x)
\end{array}\right.
$$

where $(x, \tau) \in \mathbb{R}^{n} \times \mathbb{R}^{+}, f \in C_{0}^{\infty}\left(\mathbb{R}^{n}\right)$, and $u(x, t)$ is assumed to be $C^{\infty}$ and compactly supported in the spatial variable, for simplicity. Applying the generalized Helgason Fourier transform in the spatial variable and using Proposition 7.2 we have

$$
\left\{\begin{array}{l}
\partial_{\tau} \widehat{u}(\lambda, \xi, \tau)=-\left(\lambda^{2}+\frac{(n-1)^{2}}{4 t^{2}}-\frac{n z}{t^{2}}\right) \widehat{u}(\lambda, \xi, \tau), \quad \tau>0 . \\
\widehat{u}(\lambda, \xi, 0)=\widehat{f}(\lambda, \xi)
\end{array}\right.
$$

Therefore, we obtain

$$
\widehat{u}(\lambda, \xi, \tau)=e^{-\tau\left(\lambda^{2}+\frac{(n-1)^{2}}{4 t^{2}}-\frac{n z}{t^{2}}\right)} \widehat{f}(\lambda, \xi) .
$$

Applying the inverse Helgason Fourier transform and using Corollary 7.5 we get

$$
u(x, \tau)=\left(p_{\tau} * f\right)(x)
$$

where $p_{\tau}$ is given by applying the inversion formula (52)

$$
p_{\tau}(x)=C_{n, t, z} \int_{0}^{\infty} e^{-\tau\left(\lambda^{2}+\frac{(n-1)^{2}}{4 t^{2}}-\frac{n z}{t^{2}}\right)} \Phi_{\lambda ; t}(x)|c(\lambda t)|^{-2} d \lambda .
$$

\section{A Spherical harmonics}

A spherical harmonic of degree $k \geq 0$ denoted by $Y_{k}$ is the restriction to $\mathbb{S}^{n-1}$ of a homogeneous harmonic polynomial in $\mathbb{R}^{n}$. The set of all spherical harmonics of degree $k$ is denoted by $\mathcal{H}_{k}\left(\mathbb{S}^{n-1}\right)$. This space is a finite dimensional subspace of $L^{2}\left(\mathbb{S}^{n-1}\right)$ and we have the direct sum decomposition

$$
L^{2}\left(\mathbb{S}^{n-1}\right)=\bigoplus_{k=0}^{\infty} \mathcal{H}_{k}\left(\mathbb{S}^{n-1}\right)
$$

The following integrals are obtained from the generalisation of Proposition 5.2 in [24]. 
Lemma A.1. Let $\nu \in \mathbb{C}, k \in \mathbb{N}_{0}, t \in \mathbb{R}^{+}$, and $Y_{k} \in \mathcal{H}_{k}\left(\mathbb{S}^{n-1}\right)$. Then

$$
\begin{aligned}
& \int_{\mathbb{S}^{n-1}}\left(\frac{1}{1-\frac{\langle x, \xi\rangle}{t}}\right)^{\nu} Y_{k}(\xi) d \sigma(\xi)= \\
& 2^{-k} \frac{(\nu)_{k}}{(n / 2)_{k}} \times{ }_{2} F_{1}\left(\frac{\nu+k}{2}, \frac{\nu+k+1}{2} ; k+\frac{n}{2} ; \frac{\|x\|^{2}}{t^{2}}\right) Y_{k}\left(\frac{x}{t}\right)
\end{aligned}
$$

where $x \in \mathbb{R}^{n},(\nu)_{k}$ denotes the Pochhammer symbol, and $d \sigma$ is the normalised surface measure on $\mathbb{S}^{n-1}$. In particular, when $k=0$, we have

$$
\int_{\mathbb{S}^{n-1}}\left(\frac{1}{1-\frac{\langle x, \xi\rangle}{t}}\right)^{\nu} d \sigma(\xi)={ }_{2} F_{1}\left(\frac{\nu}{2}, \frac{\nu+1}{2} ; \frac{n}{2} ; \frac{\|x\|^{2}}{t^{2}}\right) .
$$

The Gauss Hypergeometric function ${ }_{2} F_{1}$ is an analytic function for $|z|<1$ defined by

$$
{ }_{2} F_{1}(a, b ; c ; z)=\sum_{k=0}^{\infty} \frac{(a)_{k}(b)_{k}}{(c)_{k}} \frac{z^{k}}{k !}
$$

with $c \notin-\mathbb{N}_{0}$. If $\operatorname{Re}(c-a-b)>0$ and $c \notin-\mathbb{N}_{0}$ then exists the limit $\lim _{t \rightarrow 1^{-}}{ }_{2} F_{1}(a, b ; c ; t)$ and equals

$$
{ }_{2} F_{1}(a, b ; c ; 1)=\frac{\Gamma(c) \Gamma(c-a-b)}{\Gamma(c-a) \Gamma(c-b)} .
$$

Some useful properties of this function are

$$
\begin{gathered}
{ }_{2} F_{1}(a, b ; c ; z)=(1-z)^{c-a-b}{ }_{2} F_{1}(c-a, c-b ; c ; z) \\
{ }_{2} F_{1}(a, b ; c ; z)=(1-z)^{-a}{ }_{2} F_{1}\left(a, c-b ; c ; \frac{z}{z-1}\right) \\
\frac{d}{d z}{ }_{2} F_{1}(a, b ; c ; z)=\frac{a b}{c}{ }_{2} F_{1}(a+1, b+1 ; c+1 ; z) .
\end{gathered}
$$

\section{B Jacobi functions}

The classical theory of Jacobi functions involves the parameters $\alpha, \beta, \lambda \in \mathbb{C}$ (see $[13$, 14]). Here we introduce the additional parameter $t \in \mathbb{R}^{+}$since we develop our hyperbolic harmonic analysis on a ball of arbitrary radius $t$. For $\alpha, \beta, \lambda \in \mathbb{C}, t \in \mathbb{R}^{+}$, and $\alpha \neq$ $-1,-2, \ldots$, we define the Jacobi transform as

$$
\mathcal{J}_{\alpha, \beta} g(\lambda t)=\int_{0}^{+\infty} g(r) \varphi_{\lambda t}^{(\alpha, \beta)}(r) \omega_{\alpha, \beta}(r) d r
$$

for all functions $g$ defined on $\mathbb{R}^{+}$for which the integral (68) is well defined. The weight function $\omega_{\alpha, \beta}$ is given by

$$
\omega_{\alpha, \beta}(r)=(2 \sinh (r))^{2 \alpha+1}(2 \cosh (r))^{2 \beta+1}
$$

and the function $\varphi_{\lambda t}^{(\alpha, \beta)}(r)$ denotes the Jacobi function which is defined as the even $C^{\infty}$ function on $\mathbb{R}$ that equals 1 at 0 and satisfies the Jacobi differential equation

$$
\left(\frac{d^{2}}{d r^{2}}+((2 \alpha+1) \operatorname{coth}(r)+(2 \beta+1) \tanh (r)) \frac{d}{d r}+(\lambda t)^{2}+(\alpha+\beta+1)^{2}\right) \varphi_{\lambda t}^{(\alpha, \beta)}(r)=0
$$


The function $\varphi_{\lambda t}^{(\alpha, \beta)}(r)$ can be expressed as an hypergeometric function

$$
\varphi_{\lambda t}^{(\alpha, \beta)}(r)={ }_{2} F_{1}\left(\frac{\alpha+\beta+1+i \lambda t}{2}, \frac{\alpha+\beta+1-i \lambda t}{2} ; \alpha+1 ;-\sinh ^{2}(r)\right) .
$$

Since $\varphi_{\lambda t}^{(\alpha, \beta)}$ are even functions of $\lambda t \in \mathbb{C}$ then $\mathcal{J}_{\alpha, \beta} g(\lambda t)$ is an even function of $\lambda t$. Inversion formulas for the Jacobi transform and a Paley-Wiener Theorem are found in [14]. We denote by $C_{0, R}^{\infty}(\mathbb{R})$ the space of even $C^{\infty}$-functions with compact support on $\mathbb{R}$ and $\mathcal{E}$ the space of even and entire functions $g$ for which there are positive constants $A_{g}$ and $C_{g, n}, n=0,1,2, \ldots$, such that for all $\lambda \in \mathbb{C}$ and all $n=0,1,2, \ldots$

$$
|g(\lambda)| \leq C_{g, n}(1+|\lambda|)^{-n} e^{A_{g}|\operatorname{Im}(\lambda)|}
$$

where $\operatorname{Im}(\lambda)$ denotes the imaginary part of $\lambda$.

Theorem B.1. ([14],p.8) (Paley-Wiener Theorem) For all $\alpha, \beta \in \mathbb{C}$ with $\alpha \neq-1,-2, \ldots$ the Jacobi transform is bijective from $C_{0, R}^{\infty}(\mathbb{R})$ onto $\mathcal{E}$.

The Jacobi transform can be inverted under some conditions [14]. Here we only refer to the case which is used in this paper.

Theorem B.2. ([14],p.9) Let $\alpha, \beta \in \mathbb{R}$ such that $\alpha>-1, \alpha \pm \beta+1 \geq 0$. Then for every $g \in C_{0, R}^{\infty}(\mathbb{R})$ we have

$$
g(r)=\frac{1}{2 \pi} \int_{0}^{+\infty}\left(\mathcal{J}_{\alpha, \beta} g\right)(\lambda t) \varphi_{\lambda t}^{(\alpha, \beta)}(r)\left|c_{\alpha, \beta}(\lambda t)\right|^{-2} t d \lambda
$$

where $c_{\alpha, \beta}(\lambda t)$ is the Harish-Chandra c-function associated to $\mathcal{J}_{\alpha, \beta}(\lambda t)$ given by

$$
c_{\alpha, \beta}(\lambda t)=\frac{2^{\alpha+\beta+1-i \lambda t} \Gamma(\alpha+1) \Gamma(i \lambda t)}{\Gamma\left(\frac{\alpha+\beta+1+i \lambda t}{2}\right) \Gamma\left(\frac{\alpha-\beta+1+i \lambda t}{2}\right)} .
$$

This theorem provides a generalisation of Theorem 2.3 in [14] for arbitrary $t \in \mathbb{R}^{+}$. From [14] and considering $t \in \mathbb{R}^{+}$arbitrary we have the following asymptotic behavior of $\phi_{\lambda t}^{\alpha, \beta}$ for $\operatorname{Im}(\lambda)<0$ :

$$
\lim _{r \rightarrow+\infty} \varphi_{\lambda t}^{(\alpha, \beta)}(r) e^{(-i \lambda t+\alpha+\beta+1) r}=c_{\alpha, \beta}(\lambda t)
$$

Acknowledgement. The author was supported by Portuguese funds through the CIDMA - Center for Research and Development in Mathematics and Applications, and the Portuguese Foundation for Science and Technology ("FCT-Fundação para a Ciência e a Tecnologia"), within project UID/MAT/ 0416/2013.

\section{References}

[1] M. Alonso, G. Pogosyan, and K. Wolf, Wigner Functions for Curved Spaces I: On Hyperboloids, J. Math. Phys. 43 (2002), no. 12, 5857-5871.

[2] W. O. Bray, Aspects of harmonic analysis on real hyperbolic space, Fourier Analysis: Analytic and Geometric Aspects, 77-102, Lect. Notes Pure Appl. Math. vol. 157, Dekker, New York, 1994.

[3] J. L. Chen and A. A. Ungar, From the group $S L(2, \mathbb{C})$ to gyrogroups and gyrovector spaces and hyperbolic geometry, Found. Phys. 31(11) (2001), 1611-1639. 
[4] M. Ferreira, Gyrogroups in Projective Hyperbolic Clifford Analysis, I. Sabadini and F. Sommen (eds.), Hypercomplex Analysis and Applications, 61-80, Trends Math., Springer, Basel, 2010.

[5] M. Ferreira, Harmonic Analysis on the Einstein Gyrogroup, J. Geom. Symm. Phys. 35 (2014), 21-60.

[6] M. Ferreira, Harmonic Analysis on the Möbius Gyrogroup, J. Fourier Anal. Appl. 21(2) (2015), 281-317.

[7] M. Ferreira and G. Ren, Möbius Gyrogroups: A Clifford Algebra Approach, J. Algebra 328(1) (2011), 230-253.

[8] A. Grigor'yan, Heat Kernel and Analysis on Manifolds, AMS/IP Studies in Advanced Mathematics, Vol. 47, Amer. Math. Soc., Providence, RI, International Press, Boston, MA, 2009.

[9] J. Guven, Classical and quantum mechanics of a relativistic system parametrized by proper time, Phys. Rev. D (3) 4 (1991), no. 10, 3360-3363.

[10] S. Helgason, Groups and Geometric Analysis, Academic Press, Orlando FL, 1984.

[11] S. Helgason, Geometric Analysis on Symmetric Spaces, AMS, Providence, RI, 1994.

[12] S. Helgason, Non-Euclidean Analysis, A. PrÃ l'kopa and E. MolnÃąr (eds.), NonEuclidean Geometries, 367-384, Math. Appl. Ser., 581, Springer US, NY, 2006.

[13] T. Koornwinder, A New Proof of a Paley-Wiener Type Theorem for the Jacobi Transform, Ark. Mat. 13 (1975), 145-159.

[14] T. Koornwinder, Jacobi Functions and Analysis on Non-Compact Semisimple Groups, R. Askey, T. Koornwinder, and W. Schempp (eds.), Special Functions Group Theoretical Aspects and Applications, 1-84, Math. Appl. Ser., 18, Springer, Dordrecht, 1984 .

[15] C. Liu and L. Peng, Generalized Helgason-Fourier Transforms Associated to Variants of the Laplace-Beltrami Operators on the Unit Ball in $\mathbb{R}^{n}$, Indiana Univ. Math. J. 58(3) (2009), 1457-1492.

[16] W. Rudin, Function theory in the unit ball of $\mathbb{C}^{n}$, Springer-Verlag, New-York, NY, 1980 .

[17] R. S. Strichartz, Harmonic analysis as spectral theory of Laplacians, J. Funct. Anal. 87(1) (1989), 51-148.

[18] A. Terras, Harmonic Analysis on Symmetric Spaces - Euclidean Space, the Sphere, and the Poincaré Upper Half Plane, 2nd ed., Springer, New York, 2013.

[19] A.A. Ungar, Thomas Precession and the Parametrization of the Lorentz Transformation Group, Found. Phys. Lett. 1 (1988), 57-89.

[20] A.A. Ungar, Thomas Precession: its Underlying Gyrogroup Axioms and Their Use in Hyperbolic Geometry and Relativistic Physics, Found. Phys. 27(6) (1997), 881-951.

[21] A.A. Ungar, Analytic Hyperbolic Geometry - Mathematical Foundations and Applications, World Scientific, Singapore, 2005. 
[22] A.A. Ungar, The relativistic proper-velocity transformation gyrogroup, Prog. Electromagn. Res. 60 (2006), 85-94.

[23] A.A. Ungar, Analytic Hyperbolic Geometry and Albert Einstein's Special Theory of Relativity, World Scientific, Singapore, 2008.

[24] Volchkov V. V. and Volchkov Vit. V., Harmonic Analysis of Mean Periodic Functions on Symmetric Spaces and the Heisenberg Group, Springer-Verlag, London 2009. 